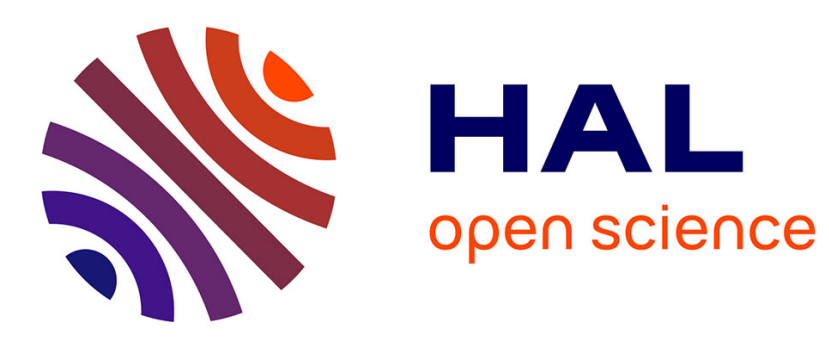

\title{
Dissipation, intermittency, and singularities in incompressible turbulent flows
}

\author{
P. Debue, V. Shukla, D. Kuzzay, D. Faranda, E.-W. Saw, F. Daviaud, B.
}

Dubrulle

\section{- To cite this version:}

P. Debue, V. Shukla, D. Kuzzay, D. Faranda, E.-W. Saw, et al.. Dissipation, intermittency, and singularities in incompressible turbulent flows. Physical Review E , 2018, 97 (5), pp.053101. 10.1103/PhysRevE.97.053101 . hal-01871198

\section{HAL Id: hal-01871198 \\ https://hal.science/hal-01871198}

Submitted on 10 Sep 2018

HAL is a multi-disciplinary open access archive for the deposit and dissemination of scientific research documents, whether they are published or not. The documents may come from teaching and research institutions in France or abroad, or from public or private research centers.
L'archive ouverte pluridisciplinaire HAL, est destinée au dépôt et à la diffusion de documents scientifiques de niveau recherche, publiés ou non, émanant des établissements d'enseignement et de recherche français ou étrangers, des laboratoires publics ou privés. 


\title{
Dissipation, intermittency, and singularities in incompressible turbulent flows
}

\author{
P. Debue, ${ }^{1}$ V. Shukla, ${ }^{1}$ D. Kuzzay, ${ }^{1,2}$ D. Faranda,${ }^{3,4}$ E.-W. Saw,${ }^{1}$ F. Daviaud,${ }^{1}$ and B. Dubrulle ${ }^{1, *}$ \\ ${ }^{1}$ DSM/IRAMIS/SPEC, CNRS UMR 3680, CEA, Université Paris-Saclay, 91190 Gif sur Yvette, France \\ ${ }^{2}$ LESIA, Observatoire de Paris, Université PSL, CNRS, Sorbonne Université, Univ. Paris Diderot, \\ Sorbonne Paris Cité, 5 place Jules Janssen, 92195 Meudon, France \\ ${ }^{3}$ DSM/LSCE, CNRS UMR 8212, CEA, Université Paris-Saclay, 91190 Gif sur Yvette, France \\ ${ }^{4}$ London Mathematical Laboratory, 14 Buckingham Street, London WC2N 6DF, United Kingdom
}

(Received 31 August 2017; revised manuscript received 28 March 2018; published 3 May 2018)

\begin{abstract}
We examine the connection between the singularities or quasisingularities in the solutions of the incompressible Navier-Stokes equation (INSE) and the local energy transfer and dissipation, in order to explore in detail how the former contributes to the phenomenon of intermittency. We do so by analyzing the velocity fields (a) measured in the experiments on the turbulent von Kármán swirling flow at high Reynolds numbers and (b) obtained from the direct numerical simulations of the INSE at a moderate resolution. To compute the local interscale energy transfer and viscous dissipation in experimental and supporting numerical data, we use the weak solution formulation generalization of the Kármán-Howarth-Monin equation. In the presence of a singularity in the velocity field, this formulation yields a nonzero dissipation (inertial dissipation) in the limit of an infinite resolution. Moreover, at finite resolutions, it provides an expression for local interscale energy transfers down to the scale where the energy is dissipated by viscosity. In the presence of a quasisingularity that is regularized by viscosity, the formulation provides the contribution to the viscous dissipation due to the presence of the quasisingularity. Therefore, our formulation provides a concrete support to the general multifractal description of the intermittency. We present the maps and statistics of the interscale energy transfer and show that the extreme events of this transfer govern the intermittency corrections and are compatible with a refined similarity hypothesis based on this transfer. We characterize the probability distribution functions of these extreme events via generalized Pareto distribution analysis and find that the widths of the tails are compatible with a similarity of the second kind. Finally, we make a connection between the topological and the statistical properties of the extreme events of the interscale energy transfer field and its multifractal properties.
\end{abstract}

DOI: 10.1103/PhysRevE.97.053101

\section{INTRODUCTION}

A defining feature of any turbulent field is the existence of fluctuations varying across a wide range of spatial and temporal scales. The shape of the energy spectrum of these fluctuations has been derived by Kolmogorov for a stationary, isotropic, homogeneous, and mirror-symmetric turbulence [1]. The starting point is the incompressible Navier-Stokes equations (INSE):

$$
\begin{aligned}
\partial_{t} u_{i}+u_{j} \partial_{j} u_{i} & =-\frac{1}{\rho} \partial_{i} p+v \partial_{j} \partial_{j} u_{i}+f_{i}, \\
\partial_{j} u_{j} & =0,
\end{aligned}
$$

where $u_{i}$ is the $d$-dimensional velocity field, $p$ the kinematic pressure, $\rho$ the density (which we set to 1 ), $f_{i}$ a $d$-dimensional forcing, and $v$ the molecular viscosity. The second moment of the INSE with the local homogeneity $(\mathrm{HH})$ assumption gives the classical Kármán-Howarth-Monin equation (hereafter KHM):

$$
\frac{1}{2} \partial_{t} E(\ell)-\epsilon=\frac{1}{4} \nabla_{\ell} \cdot\left\langle\delta \boldsymbol{u}(\delta \boldsymbol{u})^{2}\right\rangle+v \nabla_{\ell}^{2} E,
$$

where \langle\rangle denotes the statistical average, $\epsilon=\langle\boldsymbol{u} \cdot[\boldsymbol{f}(\boldsymbol{r}+$ $\ell)+f(\boldsymbol{r}-\ell)]\rangle / 2$ is a measure of the mean energy injection

\footnotetext{
*Corresponding author: berengere.dubrulle@cea.fr
}

rate, $\delta \boldsymbol{u}=\boldsymbol{u}(\boldsymbol{x}+\boldsymbol{\ell})-\boldsymbol{u}(\boldsymbol{x})$ is the velocity increments over a distance $\ell$, and $E(\ell)=\langle\boldsymbol{u}(\boldsymbol{x}) \cdot \boldsymbol{u}(\boldsymbol{x}+\boldsymbol{\ell})\rangle=\left\langle\boldsymbol{u}^{2}\right\rangle-\left\langle(\delta \boldsymbol{u})^{2}\right\rangle / 2$ is a measure of the kinetic energy at scale $\ell$. Assuming isotropy and looking for stationary self-similar solutions in the inertial range $\nu^{3 / 4} \epsilon^{-1 / 4} \ll \ell \ll L$, Kolmogorov obtained $\left\langle\delta u^{2}\right\rangle=$ $C \epsilon^{2 / 3} \ell^{2 / 3}$, or equivalently $E(k)=C_{K} \epsilon^{2 / 3} k^{-5 / 3}$, where $C_{K}$ is the Kolmogorov constant, $k$ is the wave number, and $\langle(\delta \boldsymbol{u}$. $\left.\ell / \ell)^{3}\right\rangle=-4 \epsilon \ell / 5$.

The resulting standard phenomenological description (K41) is that of a cascade process, in which the energy driving the flow, injected at the large length scales $\ell \sim L$ comparable to the system size, is transferred to the smaller scales in a self-similar manner down to the Kolmogorov length scale $\eta=v^{3 / 4} \epsilon^{-1 / 4}$, where it is dissipated by the viscous processes. In a steady state, the mean rate of energy injection equals the inter-length-scale energy transfer rate at each scale down to the Kolmogorov scale, where it becomes the viscous energy dissipation $\epsilon$. This self-similar picture was challenged by Landau, as one of the basic assumptions of the $\mathrm{K} 41$, namely that $\epsilon$ is constant, is flawed. Measurements show that it exhibits short bursts in the time series or intense fluctuations over localized regions. The breaking of exact self-similarity was subsequently confirmed by the scaling properties of the velocity structure functions $S_{p}=\left\langle[\delta u(x, \ell)]^{p}\right\rangle$, which deviate from the self-similar law $S_{p} \sim \ell^{p / 3}$. This led Kolmogorov to formulate in 1962 a refined scaling hypothesis $[2,3]$ to bridge the large-scale and small- 
scale behavior using the quantity $\epsilon_{\ell}$, characterizing viscous dissipation averaged over a ball of size $\ell$, as

$$
\epsilon_{\ell} \sim \frac{[\delta u(x, \ell)]^{3}}{\ell} .
$$

This results in $S_{p}=\left\langle\epsilon_{\ell}^{p / 3}\right\rangle \ell^{p / 3} \sim \ell^{\tau(p)+p / 3} \sim \ell^{\zeta(p)}$, showing that all the corrections to self-similarity are given by the statistics of $\epsilon_{\ell}$, via the function $\tau(p)$.

However, Kraichnan [4] suggested that if such a relation holds, then $\epsilon_{\ell}$ should represent some well-defined local energy flux $\Pi_{\ell}$ rather than a local energy dissipation averaged over a volume of linear dimension $\ell$. The problem of defining the local subscale-energy-flux was considered by Meneveau [5] by employing wavelets to measure the energy transfer to scales smaller than $\ell$ at any space point $\boldsymbol{x}$, but this analysis was shown to be flawed [6]. Eyink used the coarse-graining approach to obtain a local energy balance in space and scale from the INSE and thereby was able to identify the local energy flux from the large scales to the small scales as

$$
\begin{aligned}
\Pi_{\ell}(\boldsymbol{x}) & \equiv-\operatorname{Tr}\left[\left(\nabla \boldsymbol{u}^{\ell}\right) \boldsymbol{\tau}^{\ell}\right], \\
\tau_{i j}^{\ell} & =\left(u_{i} u_{j}\right)^{\ell}-u_{i}^{\ell} u_{j}^{\ell},
\end{aligned}
$$

where the superscript refers to a coarse-grained field at scale $\ell$ and $\tau$ represents a stress tensor (because of the small-scale components $<\ell$ ). Physically we can view $\Pi_{\ell}(\boldsymbol{x})$ as the effective dissipation of the energy contained in the scales larger than $\ell$ by the action of the stress, coming from scales smaller than $\ell$, on the gradients of the large-scale motion [6].

As discussed in Ref. [6], the above definition guarantees that whenever $\delta u(x, \ell)=O\left(\ell^{h}\right)$, then $\Pi_{\ell}=O\left(\ell^{3 h-1}\right)$. Such a formulation then allows us to link the occurrence of intermittency to the local scaling and regularity properties of the velocity field. Indeed, we find that if $h>1 / 3, \Pi_{\ell}$ vanishes as $\ell \rightarrow 0$ and the weak solutions to the Euler equations do conserve energy; conversely, if $h<1 / 3$, then there may exist solutions which dissipate energy, i.e., even in the absence of viscosity $v=0$. This behavior of the weak solutions was conjectured by Onsager in 1949 [7]. Now, if such a solution starts to develop in a viscous fluid, it will be regularized at a scale $\eta_{h} \propto v^{1 /(1+h)}$, at which $h$ becomes 1 . We refer to this behavior of the solution as an instance of a quasisingularity. The viscous dissipation becomes dominant and constant below $\eta_{h}$, while the energy flux vanishes like $\ell^{2}$. For $h \leqslant 1 / 3$, this results in a "spot" of large energy dissipation at the location of the quasisingularity and this explains the intermittency.

Another way of interpreting the above behavior is at the probabilistic level, in which case we postulate that the solutions of the INSE obey the scaling $\delta u(x, \ell) \sim \ell^{h}$ only in a statistical sense [8]. The probability of occurrence of such an event then varies as $P(h, \ell) \sim \ell^{C(h)}$ [8]. In Ref. [9] Eyink used a simple Borel-Cantelli argument to show that such a statistical interpretation implies, almost with certainty, that the velocity field realizations are singular. Moreover, a precise connection between structure function scaling exponents and intermittency can be made by using the multifractal formalism, i.e., in the form of the spatial dimension of the singularity set by means of an explicit variational formula [10]. The latter yields $\tau(p)=\min _{h}[p(3 h-1)+C(h)]$, whereas in the deterministic interpretation, $C(h)$ has the meaning of the codimension of the set of points where a quasisingularity of exponent $h$ occurs.

Irrespective of the interpretation, we see that to understand intermittency, it is interesting to study the local energy transfer $\Pi_{\ell}(\boldsymbol{x})$. However, a closer examination of the expression for the local flux in Eq. (3) shows that it is a sum of products of two terms: (a) $\nabla_{j} u_{i}^{\ell}=O\left(\ell^{h-1}\right)$ and (b) $\tau_{i j}^{\ell}=O\left(\ell^{2 h}\right)$. Therefore, if we take the case of $h=1 / 3$ as an illustrative example, then in the limit $\ell \rightarrow 0$ the term (a) is unbounded and the term (b) goes to zero smoothly. So, even though the product is theoretically bounded, it is very likely that any attempt to determine $\Pi_{\ell}$ at small scales by using the data from experiments will be prone to unavoidable noise issues, thereby resulting in values which are either too large or small compared to the exact value.

In this paper, we discuss another expression of the local flux, which is devoid of the above-mentioned potential flaw. A weak formulation has been used to derive the local energy balance equation for the three-dimensional (3D) incompressible Euler and NS equations in Ref. [11]. This local energy balance is similar to the classical Kármán-Howarth-Monin (KHM) relation, but it is local in space and does not require the velocity field to be homogeneous or regular. It describes the temporal evolution of the point-split kinetic energy at a given scale $\ell$ and position $\boldsymbol{x}$. It has three main constituent terms: (1) a spatial flux term, describing how the input energy is transported within the flow; (2) a local energy transfer $\mathscr{D}_{\ell}^{I}$ describing how the energy is transferred locally through scales by nonlinear interactions; (3) a term describing the energy transfer and dissipation by viscosity. The $\ell \rightarrow 0$ limit of $\mathscr{D}_{\ell}^{\mathrm{I}}$ gives the contribution to the local dissipation stemming from the eventual lack of smoothness in the solution (singularities), also known as inertial dissipation, $\mathscr{D}^{\mathrm{I}}$.

Such a generalization of the classical KHM relation allows us to handle possible singularities and quasisingularities and to study their impact on the energy transfer and dissipation; it also puts Kolmogorov's 1962 refined similarity hypothesis in a natural framework. This motivates us to explore the statistics of the interscale flux and viscous terms in a fully developed turbulence as the scale $\ell$ is varied from in the inertial range down to the dissipation scale and analyze how they relate potential singularities or quasisingularities with intermittency.

In the present paper, we perform an experimental study of the connection between the local energy flux and intermittency by analyzing the velocity fields measured via stereo particle image velocimetry (SPIV) in the turbulent von Kármán swirling flow. We generate turbulence at very high Reynolds numbers in a cylindrical vessel filled with fluid by using two independently rotating impellers. Our experimental setup allows us to perform experiments for a long duration of time (ranging from minutes to hours), so as to accumulate sufficient statistics for a reliable data analysis. We also perform direct numerical simulations of the INSE at a moderate resolution to compare with the trends from the analysis of the experimental data.

We connect the high-order statistics of the interscale transfer with the statistics of the velocity field and show that they are compatible with the refined similarity hypothesis. We condition the velocity structure functions on the regions of large or small inertial dissipation and show that the intermittency corrections are governed by the extreme events of the inertial 
dissipation. Furthermore, we characterize the statistics of these extreme events via generalized Pareto distribution analysis (a peak over threshold approach) and discuss its link with the multifractal analysis.

In Sec. II we give a brief overview of the mathematical background and present details of the experimental setup and the numerical simulations. We present the results of our study in Sec. III and provide a discussion and the conclusions in Sec. IV.

\section{METHODS}

\section{A. Mathematical background}

In this section we briefly review the mathematical framework which we use to analyze the data from the experiments and the supporting numerical simulations. In our discussion we closely follow the material in Refs. [11,12]. We consider a weak solution $\boldsymbol{u}$ of the NS equation Eq. (1a) in the absence of forcing, i.e., $f=0$, to keep the analysis simple. We now introduce a regularized velocity field

$$
\boldsymbol{u}^{\ell}(\boldsymbol{x}, t)=\phi^{\ell} * \boldsymbol{u}(\boldsymbol{x}, t),
$$

where

$$
\phi^{\ell}(\xi)=\frac{1}{\ell^{3}} \phi(\xi / \ell)
$$

is an infinitely differentiable function with compact support on $\mathbb{R}^{3}$, even, non-negative with integral one. The regularized velocity field obeys regularized NS equations given by

$$
\begin{aligned}
\partial_{t} u_{i}^{\ell}+\partial_{j}\left(u_{i} u_{j}\right)^{\ell} & =-\partial_{i} p^{\ell}+v \partial_{k} \partial_{k} u_{i}^{\ell}, \\
\partial_{j} u_{j}^{\ell} & =0 .
\end{aligned}
$$

We remark that we can regard $\boldsymbol{u}^{\ell}$ as a continuous wavelet transform of the velocity field $\boldsymbol{u}$ with respect to the wavelet $\phi$, provided the latter has the correct properties [13].

We multiply Eq. (1a) and Eq. (6a) by $\boldsymbol{u}^{\ell}$ and $\boldsymbol{u}$, respectively, and add them together; after the rearrangement of terms we obtain the following balance equation:

$$
\begin{aligned}
\partial_{t} u_{i} u_{i}^{\ell}+\partial_{i} T_{i} & =\mathcal{E}^{\ell}-2 v \partial_{j} u_{i}^{\ell} \partial_{j} u_{i}, \\
T_{i} & =u_{j} u_{j}^{\ell} u_{i}+p^{\ell} u_{i}+p u_{i}^{\ell}-v \partial_{i} u_{j} u_{j}^{\ell}, \\
\mathcal{E}^{\ell} & =u_{i} u_{j} \partial_{i} u_{j}^{\ell}-\partial_{i}\left(u_{i} u_{j}\right)^{\ell} u_{j} .
\end{aligned}
$$

We rewrite $\mathcal{E}^{\ell}$ as

$$
\begin{aligned}
-2 \mathcal{E}^{\ell}= & \int \nabla \phi^{\ell}(\xi) \cdot \delta \boldsymbol{u}(\delta \boldsymbol{u})^{2} d \xi-\partial_{i}\left(u_{i} u_{j} u_{j}\right)^{\ell} \\
& +\partial_{i}\left[u_{i}\left(u_{j} u_{j}\right)^{\ell}\right],
\end{aligned}
$$

where $\delta \boldsymbol{u}$ is the velocity increment over a distance $\xi$ and $\boldsymbol{\nabla}$ the gradient over $\boldsymbol{\xi}$ [11]. Also,

$$
\begin{aligned}
v \partial_{j} u_{i}^{\ell} \partial_{j} u_{i}= & -v \int \nabla^{2} \phi^{\ell}(\xi) u_{i}(\boldsymbol{x}) u_{i}(\boldsymbol{x}+\boldsymbol{\xi}) d \xi \\
& -v \partial_{j} \int \nabla_{j} \phi^{\ell}(\xi) u_{i}(\boldsymbol{x}) u_{i}(\boldsymbol{x}+\boldsymbol{\xi}) d \xi .
\end{aligned}
$$

Finally, we note that $2 E^{\ell}(\boldsymbol{x}) \equiv u_{i} u_{i}^{\ell}=\int \phi^{\ell}(\boldsymbol{\xi}) u_{i}(\boldsymbol{x}) u_{i}(\boldsymbol{x}+$ $\xi) d \xi$. Therefore, we have

$$
\begin{aligned}
\partial_{t} E^{\ell}(\boldsymbol{x})+\partial_{j} J_{j}= & \frac{1}{4} \int \nabla \phi^{\ell}(\xi) \cdot \delta \boldsymbol{u}(\delta \boldsymbol{u})^{2} d \boldsymbol{\xi} \\
& +v \int \nabla^{2} \phi^{\ell}(\xi) \boldsymbol{u}(\boldsymbol{x}) \cdot \boldsymbol{u}(\boldsymbol{x}+\boldsymbol{\xi}) d \boldsymbol{\xi} \\
\equiv & -\mathscr{D}_{\ell}^{\mathrm{I}}-\mathscr{D}_{\ell}^{v},
\end{aligned}
$$

where

$$
\begin{aligned}
J_{i}= & u_{i} E^{\ell}+\frac{1}{2}\left(p^{\ell} u_{i}+p u_{i}^{\ell}\right) \\
& -\frac{1}{4}\left[\left(u_{i} u_{j} u_{j}\right)^{\ell}-u_{i}\left(u_{j} u_{j}\right)^{\ell}\right]-v \partial_{i} E^{\ell} \\
& +v \int \nabla_{i} \phi^{\ell}(\xi) u_{j}(\boldsymbol{x}) u_{j}(\boldsymbol{x}+\boldsymbol{\xi}) d \xi .
\end{aligned}
$$

Equation (10) is a local nonrandom form of the classical KHM equation and describes the evolution of the point-split kinetic energy at scale $\ell$ and at position $\boldsymbol{x}$ through three main ingredients: (1) a spatial flux term $\boldsymbol{\nabla} \cdot \boldsymbol{J}$, which describes how the input energy is transported within the flow; (2) an interscale flux $\mathscr{D}_{\ell}^{\mathrm{I}}$, which describes how the energy cascades locally across the length scales; and (3) $\mathscr{D}_{\ell}^{v}$, which describes space transfer and dissipation of energy by viscosity. Note that the main assumption in the derivation of the weak KármánHowarth-Monin (WKH) equation, namely the existence of a singularity or quasisingularity, breaks the homogeneity assumption; however, if the singularities fluctuate in time and space, homogeneity may be recovered, albeit in a statistical sense [8].

The contribution to the local dissipation because of the possible lack of smoothness of the velocity field is given by

$$
\mathscr{D}^{\mathrm{I}}=\lim _{\ell \rightarrow 0}\left(\lim _{\nu \rightarrow 0} \mathscr{D}_{\ell}^{\mathrm{I}}\right) ;
$$

note that the order of limits is important: we first take the high Reynolds number limit $v \rightarrow 0$ and then $\ell \rightarrow 0$. The sign of $\mathscr{D}^{\mathrm{I}}$ depends on the space dimension. In one dimension, for the Burgers equation $\mathscr{D}^{\mathrm{I}} \geqslant 0$. In two dimensions $\mathscr{D}^{\mathrm{I}}=0$. In $3 \mathrm{D}$, the condition of existence of a suitable weak solution only implies that the spatial average $\left\langle\mathscr{D}^{\mathrm{I}}\right\rangle \geqslant 0$, while the instantaneous local $\mathscr{D}^{\mathrm{I}}$ depends on the regularity of the velocity field. It is zero if the velocity field is regular and nonzero in the presence of a singularity of the Euler equation. As long as $h<1 / 3$, the local energy transfer, which scales like $\ell^{3 h-1}$, is prone to increase locally with decrease in the scale $\ell$ until reaching $\ell=\eta_{h}$ where it is dissipated. This results in a huge variation of the local energy dissipation and intermittency [14]. Such a scenario has already been validated on the shell models of turbulence [15]. This suggests that a natural analog of the quantity $\epsilon_{\ell}$ is $\mathscr{D}_{\ell}^{\mathrm{I}}$ [14]. In the present paper we show conclusively by making use of the analytical, numerical, and experimental analysis that it is indeed the case.

\section{B. Experimental setup and the description of the flow field}

We use the von Kármán flow setup at SPEC to conduct experiments for this study. Here we briefly describe the experimental setup and refer to Appendix A 1 and Refs. [16,17] 
TABLE I. Parameters describing the five experimental data sets (A, B, C, D, and E) and the DNS run. $F$ is the rotation frequency of the impellers in $\mathrm{Hz}$; $\mathrm{Re}$ is the Reynolds number based on the radius of the tank; $\operatorname{Re}_{\lambda}$ is the Taylor-microscale Reynolds number; $\epsilon$ is the dimensionless energy dissipation, reported in Ref. [17] for the data sets A-E; $\eta$ is the Kolmogorov dissipation length scale; and $\Delta x$ represents the spatial resolution in the measurements and the DNS. The second to last column, Samples, indicates the number of velocity field samples over which the statistical averaging is performed (product of number of time frames and number of spatial points), and the last column shows the symbols used to represent the experimental data sets.

\begin{tabular}{|c|c|c|c|c|c|c|c|c|c|}
\hline Case & $F(\mathrm{~Hz})$ & Glycerol content & $\operatorname{Re}$ & $\operatorname{Re}_{\lambda}$ & $\epsilon$ & $\eta(\mathrm{mm})$ & $\Delta x(\mathrm{~mm})$ & Samples & Symbol \\
\hline A & 5 & $0 \%$ & $3 \times 10^{5}$ & 1870 & 0.0254 & 0.02 & 2.4 & $29999 \times 89 \times 65$ & $\circ$ \\
\hline $\mathrm{B}$ & 5 & $0 \%$ & $3 \times 10^{5}$ & 2750 & 0.0450 & 0.02 & 0.48 & $29228 \times 77 \times 79$ & $\square$ \\
\hline $\mathrm{C}$ & 5 & $0 \%$ & $3 \times 10^{5}$ & 2510 & 0.0502 & 0.02 & 0.24 & $28000 \times 162 \times 157$ & $\diamond$ \\
\hline $\mathrm{D}$ & 1 & $0 \%$ & $4 \times 10^{4}$ & 917 & 0.0413 & 0.08 & 0.48 & $9999 \times 77 \times 80$ & $\triangle$ \\
\hline $\mathrm{E}$ & 1.2 & $59 \%$ & $6 \times 10^{3}$ & 214 & 0.0275 & 0.37 & 0.24 & $30188 \times 151 \times 174$ & $\star$ \\
\hline DNS & & $512^{3}$ & & 138 & 0.0182 & 0.0091 & $2 \eta / 3$ & $24 \times 512^{3}$ & \\
\hline
\end{tabular}

for more details. The setup consists of a cylindrical tank of height $H=20 \mathrm{~cm}$ and radius $R=10 \mathrm{~cm}$ to hold the test fluid. We drive the fluid to and maintain it in a turbulent state at high Reynolds numbers by means of two independently rotating impellers at frequency $F$, located at the top and the bottom of the cylindrical tank. We measure the radial $u_{\mathrm{r}}$, axial $u_{\mathrm{z}}$, and azimuthal $u_{\phi}$ components of the velocity fields by using high-zooming lenses coupled to a standard particle image velocimetry (SPIV) technique. We perform our measurements within a $4 \mathrm{~cm} \times 3 \mathrm{~cm}$ region located on the meridian plane, around the symmetry point of the experimental setup (see Fig. 16 in Appendix A2). At this location, a shear layer induced by the differential rotation produces a strong turbulent motion $[18,19]$. We used the SPIV data from these experiments in Ref. [17] to present an extensive characterization of the intermittency by using the velocity structure functions in such a flow configuration. However, in this study our goal is more fundamental and we want to trace the origin of the intermittency corrections and connect them with extreme events of local dissipation $\mathscr{D}_{\ell}^{\mathrm{I}}$ and $\mathscr{D}_{\ell}^{v}$.

The Reynolds number based on the radius of the tank is $\mathrm{Re}=2 \pi R^{2} F / \nu$, where $\nu$ is the kinematic viscosity of the fluid in the tank. The local energy injection rate $\epsilon$ has been computed in [17]. In our experiments, we use a mixture of water and glycerol and by varying their proportion we can control the viscosity of the fluid; this in turn allows us to tune the Kolmogorov scale $\eta$. This feature coupled with the use of the multiscale imaging method in our experiments enables us to access scales in the range of $\eta$ to $5000 \eta$ (i.e., three decades of inertial range) in a fully turbulent flow. Table I summarizes the parameters corresponding to the different data sets used throughout this paper.

\section{Direct numerical simulations}

In our analysis of the experimental data, we check for the influence of experimental errors, anisotropy, inhomogeneity, finite boundaries, and projection effects (our measurements are on a plane and not over a volume). We implement these checks by comparing the results from the analysis of the experimental with those from the direct numerical simulations (DNS) of the 3D NS Eq. (1a). To carry out the DNS, we use the NSE solver VIKSHOBHA, an efficient, parallel numerical code based on a pseudospectral method. We use this to determine the fluid velocity $\boldsymbol{u}$ on a cubic, triply periodic domain with sides $L=$ $L_{\mathrm{x}}=L_{\mathrm{y}}=L_{\mathrm{z}}=2 \pi$.

The use of the periodic boundary conditions allow us to express $\boldsymbol{u}$ as a Fourier series. However, in any DNS only a finite number of Fourier modes can be used. Therefore, to limit the number of Fourier modes, we make use of a Galerkin truncation and solve the NSE in Fourier space with $N_{\mathrm{c}}^{3}$ modes. The velocity field is now given by $\boldsymbol{u}(\boldsymbol{x}, t)=\Sigma_{\boldsymbol{k}} \hat{\boldsymbol{u}}_{\boldsymbol{k}} \exp [i \boldsymbol{k} \cdot \boldsymbol{x}]$, where $\boldsymbol{k}=\left(n_{1}, n_{2}, n_{3}\right) 2 \pi / L$ and $n_{\mathrm{i}} \in\left[-N_{\mathrm{c}}, N_{\mathrm{c}}-1\right]$. In other words, we discretize the computational domain and use an $N_{\mathrm{c}}^{3}$ number of grid points to represent $\boldsymbol{u}$ in the real (physical) space. We use the incompressibility condition $\boldsymbol{\nabla} \cdot \boldsymbol{u}$ to eliminate the pressure by introducing a transverse projection operator $\mathbb{P}_{\mathrm{i}, \mathrm{k}}(\boldsymbol{k})=\delta_{\mathrm{i}, \mathrm{j}}-k_{\mathrm{i}} k_{\mathrm{j}} / k^{2}$, which projects the nonlinear term $\boldsymbol{u} \cdot \boldsymbol{\nabla} \boldsymbol{u}$ on the plane perpendicular to $\boldsymbol{k}$. To implement the pseudospectral method, we compute the linear terms in Fourier space and the nonlinear term in real space, which we then transform to Fourier space; to remove aliasing errors we use a 2/3-dealiasing rule, so that the maximum wave number in our simulations is $k_{\max }=N_{\mathrm{c}} / 3$. We evolve the NS equations in time by using a second-order Runge-Kutta scheme, while exactly solving the linear part.

In the present work, we take $N_{\mathrm{c}}=512$ and use the TaylorGreen forcing at wave number $\tilde{k}_{f}=1$ with an amplitude $f_{0}=0.12$ (see Appendix A 7 for more details). We use several independent velocity samples to compute the same diagnostics as for the experimental flow field, but now in a 3D volume rather than on a plane.

\section{RESULTS}

We now present the results from our analysis of the experimental flow field and the numerical simulations.

\section{A. Wavelet structure functions}

The local energy transfers are computed by using the wavelet transforms. Therefore, it is natural to make a link between the intermittency of the velocity field and the local energy transfer via the wavelet structure functions, rather than the classical structure functions. They are defined by using the 

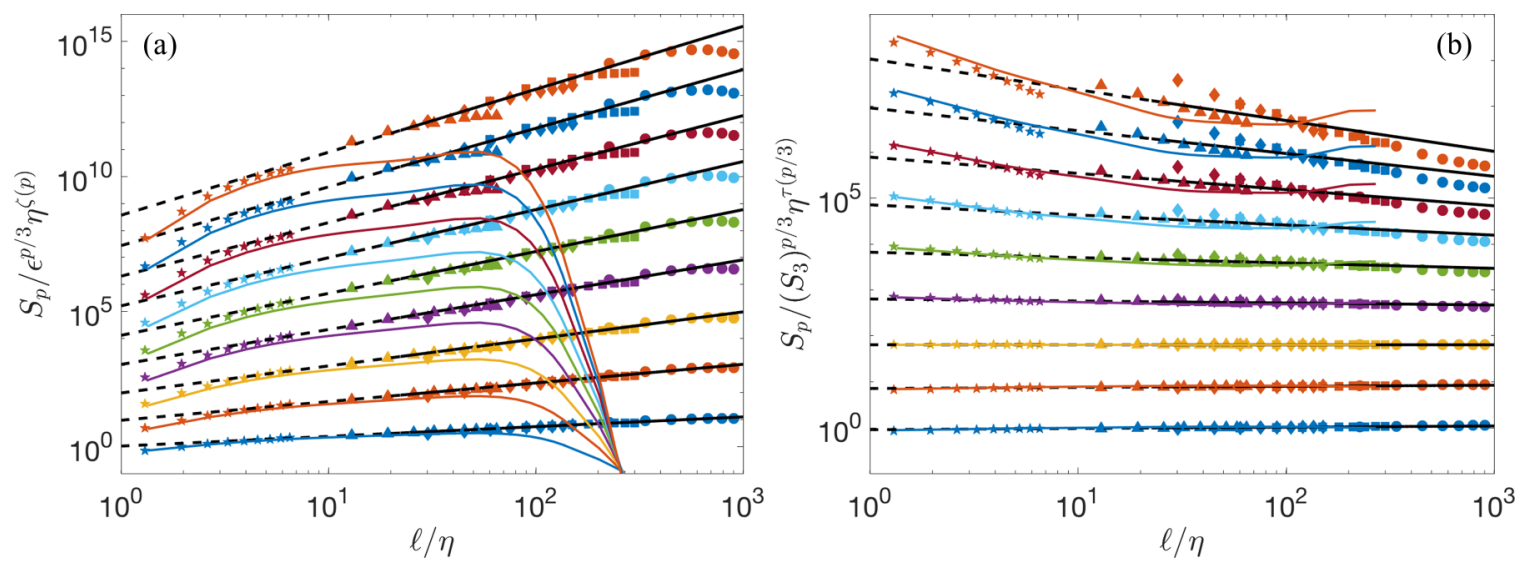

FIG. 1. Wavelet structure functions. (a) Plots of $S_{p} / \epsilon^{p / 3} \eta^{\zeta(p)}$ vs $\ell / \eta$ for the first nine orders: $p=1$ (light blue), $p=2$ (orange), $p=3$ (yellow), $p=4$ (purple), $p=5$ (green), $p=6$ (pale blue), $p=7$ (red), $p=8$ (strong blue), and $p=9$ (deep orange). The black dashed lines indicate the power-law behavior $(\ell / \eta)^{\zeta(p)}$ [see Table II for the values of $\zeta(p)$ ]. (b) Plots of the rescaled structure functions $S_{p} / S_{3}^{p / 3}$ vs $\ell / \eta$. The black dashed lines indicate the power law $(\ell / \eta)^{\zeta(p)-p / 3} . \eta$ is the Kolmogorov dissipation length scale and $\epsilon$ is the dimensionless energy injection rate. The structure function of any order $p$ is obtained by combining the five different experimental data sets, shown here by using different symbols: A (circles), B (squares), C (diamonds), D (triangles), and E (stars). A comparison with the DNS results is shown by plotting $S_{p}$ of different orders as solid curves with the same color scheme as used for the experimental data sets. The structure functions have been shifted by multiplying with an arbitrary factor for visual clarity.

following quantities:

$$
\begin{aligned}
G_{i j}(\boldsymbol{x}, \ell) & =\int d \boldsymbol{y} \boldsymbol{\nabla}_{j} \Phi_{\ell}(\boldsymbol{y}) u_{i}(\boldsymbol{x}+\boldsymbol{y}), \\
S_{i j}(\boldsymbol{x}, \ell) & =\frac{1}{2}\left[G_{i j}(\boldsymbol{x}, \ell)+G_{j i}(\boldsymbol{x}, \ell)\right], \\
A_{i j}(\boldsymbol{x}, \ell) & =\frac{1}{2}\left[G_{i j}(\boldsymbol{x}, \ell)-G_{j i}(\boldsymbol{x}, \ell)\right],
\end{aligned}
$$

where $\Phi_{\ell}(x)=\ell^{-d} \Phi(x / \ell)$ is a Gaussian function and $d$ is the space dimension. We then compute the wavelet velocity increments as

$$
\delta W(\boldsymbol{u})(\boldsymbol{x}, \ell)=\left(\delta_{L}^{2}+\delta_{\perp}^{2}\right)^{1 / 2},
$$

where

$$
\begin{aligned}
& \delta_{L}(\ell)=\ell \max _{i j}\left|S_{i j}(\boldsymbol{x}, \ell)\right|, \\
& \delta_{\perp}(\ell)=\ell \max _{i j}\left|A_{i j}(\boldsymbol{x}, \ell)\right|,
\end{aligned}
$$

respectively. We use the increments $\delta W(\boldsymbol{u})(\boldsymbol{x}, \ell)$ to infer the general scaling properties of the velocity fields by defining the structure functions as

$$
S_{p}(\ell)=\left\langle|\delta W(\boldsymbol{u})(\boldsymbol{x}, \ell)|^{p}\right\rangle,
$$

where \langle\rangle denotes an average over space and time. This way of defining the structure functions has some similarities with those based on the principal values of $\left|G_{i j}^{R}(\boldsymbol{x})\right|$ as reported by Kestener and Arneodo in Ref. [20]. Like in Ref. [20], our approach does not involve the derivatives of the velocity field, nor does it introduce any additional noise. Moreover, our definition is free of singular values computation, a procedure which may generate some noise. Therefore, ours is the smoothest possible definition of the structure functions, which enables us to quantify the scaling properties of a given velocity field.

We first check that this definition provides a description of the intermittency that is compatible with what is obtained by using the classical velocity increments. In Fig. 1(a) we plot the structure functions $S_{p}(\ell), p \in(1,9)$ for the five experimental flows A to E (see Table I for more details). We observe that the plots of $S_{p}(\ell)$ rescaled by $\epsilon^{p / 3} \eta^{\zeta(p)}$ vs $\ell / \eta$ collapse on a universal curve for the cases $\mathrm{A}$ to $\mathrm{E}$, which are different for different $p$. In Table II we summarize the exponents $\zeta(p)$, which we obtain for the different orders of $S_{p}(\ell)$. The scaling behavior is similar to what is observed for the structure functions computed by directly using velocity increments [17]. Also, the scaling exponents are in good agreement with those computed by using extended self-similarity [21]. Moreover, we find that these structure functions compare well with those computed from the DNS velocity field; in particular, we have a good agreement with the experimental data set $\mathrm{E}$, which has similar $\operatorname{Re}_{\lambda}$.

We note that the structure functions from different experimental data sets deviate significantly, by being systematically lower, from those at higher $\operatorname{Re}_{\lambda}$. In [17], this difference between

TABLE II. Values of the multiscaling exponents $\zeta(p), \tau(p)$, and $\gamma(p)$ for the velocity, the local transfers, and viscous dissipation structure functions, respectively, of different orders (see text for more details). The values of $\zeta(p)$ listed here have been taken from Ref. [17].

\begin{tabular}{lcccccrrrr}
\hline \hline Exponent/Order $p$ & 1 & 2 & 3 & 4 & 5 & 6 & 7 & 8 & 9 \\
\hline$\zeta(p)$ & 0.36 & 0.69 & 1 & 1.29 & 1.55 & 1.78 & 1.98 & 2.17 \\
$\tau(p / 3)$ & 0.03 & 0.02 & 0 & -0.04 & -0.12 & -0.22 & -0.30 & -0.50 & -0.67 \\
$\gamma(p / 3)$ & 0.01 & 0.01 & 0 & -0.02 & -0.06 & -0.10 & -0.16 & -0.22 & -0.30 \\
\hline \hline
\end{tabular}



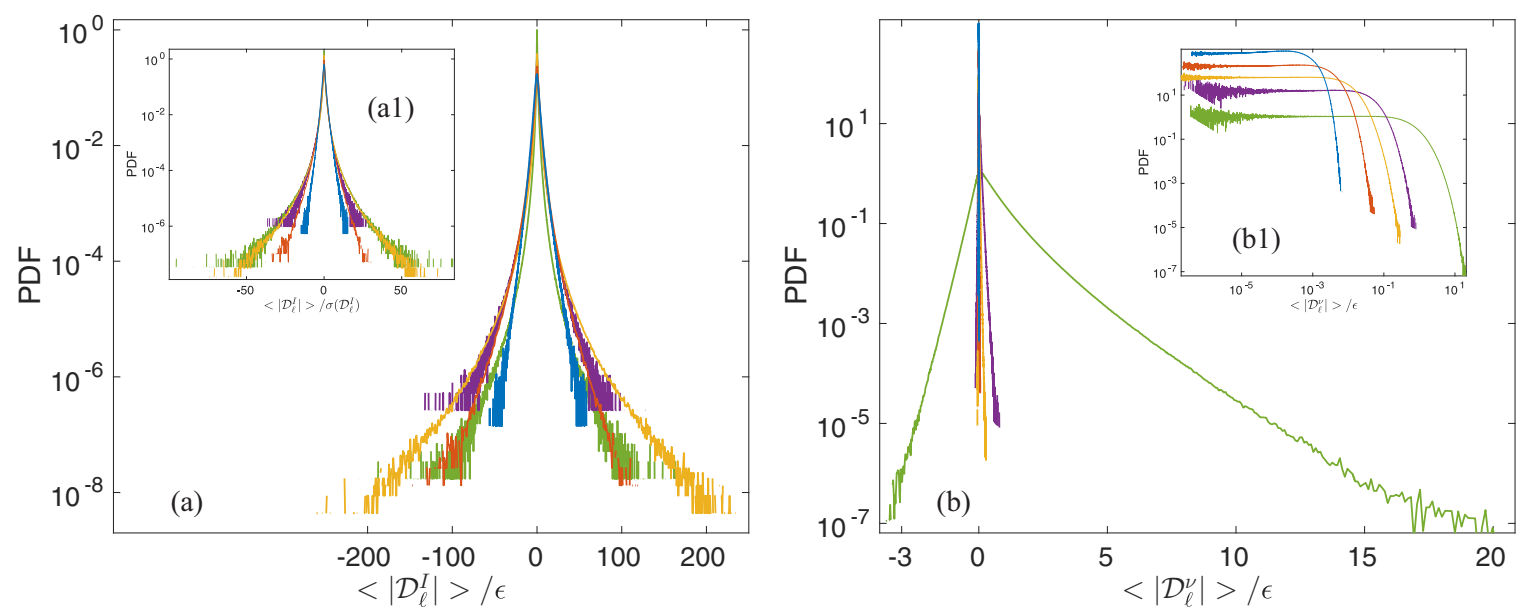

FIG. 2. Plots of the probability distribution function of the instantaneous values of the (a) local energy transfer $\mathscr{D}_{\ell}^{\mathrm{I}} / \epsilon$ and (b) viscous dissipation $\mathscr{D}_{\ell}^{v}$ at different scales. The PDFs at the different scales are shown by using different colors: $\ell / \eta=680$ blue curve (data set A), $\ell / \eta=240$ red curve (data set $\mathrm{B}), \ell / \eta=90$ yellow curve (data set $\mathrm{C}), \ell / \eta=30$ purple curve (data set $\mathrm{D})$, and $\ell / \eta=3$ green curve (data set $\mathrm{E}$ ). $\eta$ is the Kolmogorov dissipation length scale and $\epsilon$ is the dimensionless energy injection rate. Inset (a1): Same as in panel (a) but $\mathscr{D}_{\ell}^{\mathrm{I}}$ normalized with the standard deviation of the distribution. Inset (b1): A log-log plot of the PDF of viscous dissipation $\mathscr{D}_{\ell}^{v}$ at different scales for the positive values only.

different experimental cases was attributed to inhomogeneities of the dissipation. A way to test this hypothesis is to consider rescaled wavelet structure functions, i.e., $S_{p}(\ell) /\left[S_{1}(\ell)\right]^{n / 3}$, since in such a case the dependence with respect to $\epsilon$ cancels. We plot these rescaled structure functions in Fig. 1(b) and find an improvement in their collapse on the universal curves for all the data sets A to E. Now they follow the expected scaling law $\ell^{\tau(p / 3)}$, which is compatible with the scaling $S_{3}(\ell) \sim \ell$. Also, the agreement with the control DNS is also improved.

\section{B. Spatiotemporal statistics of the local energy transfer and viscous dissipation}

We now explore the connection between the intermittency study presented above and the statistics of the local energy transfer and viscous dissipation. We compute $\mathscr{D}_{\ell}^{\mathrm{I}}$ and $\mathscr{D}_{\ell}^{v}$ for the experimental data sets A to E. Note that in our experimental setup, the velocity fields are such that their fluctuations have spatially homogeneous statistics; they do not depend on space.

\section{Probability distribution functions}

In Figs. 2(a) and 2(b) we show the probability distribution functions (PDFs) of the local interscale transfer $\mathscr{D}_{\ell}^{\mathrm{I}}$ and the viscous dissipation $\mathscr{D}_{\ell}^{v}$, respectively, at different scales, by accumulating data from the instantaneous fields. Both quantities are very intermittent in space and time. $\mathscr{D}_{\ell}^{v}$ is predominantly positive; the percentage of the positive values varies between $67 \%$ and $90 \%$, as we move from the large length scales to small length scales close to $\eta$, respectively. The existence of negative values is due to the fact that $\mathscr{D}_{\ell}^{v}$ is the sum of a transport term and a dissipation term, both originating from the viscous part [see Eq. (9)]. The relative importance of the latter increases with the decreasing scales. This also explains the difference with the traditional viscous dissipation term $\epsilon_{v}=v \partial_{i} u_{j} \partial_{i} u_{j}$ (see Fig. 3), whose PDF is close to the log-normal form. In the inset Fig. 2(b1) we plot the PDFs of the positive values of
$\mathscr{D}_{\ell}^{v}$, which show a clear impact of the viscous dissipation at the smaller scales. In contrast, the PDFs of $\mathscr{D}_{\ell}^{\mathrm{I}}$ are rather symmetric with both large positive and negative values representing direct and inverse (backscatter) energy transfer, respectively. These PDFs display wide tails, irrespective of whether we choose the total dissipation $\epsilon$ or the standard deviation of $\mathscr{D}_{\ell}^{v}$ to normalize $\mathscr{D}_{\ell}^{v}$. The tail width of the PDFs of $\mathscr{D}_{\ell}^{\mathrm{I}} / \epsilon$ initially increases and then decreases, as we move from large to small scales. If $\mathscr{D}_{\ell}^{\mathrm{I}}$ is normalized by its standard deviation, we observe that the decrease of the tail width of the PDFs is less pronounced at small scales; this results in the overlap of the PDF tails for the length scales $90 \eta$ and $3 \eta$. This suggests that the behavior of

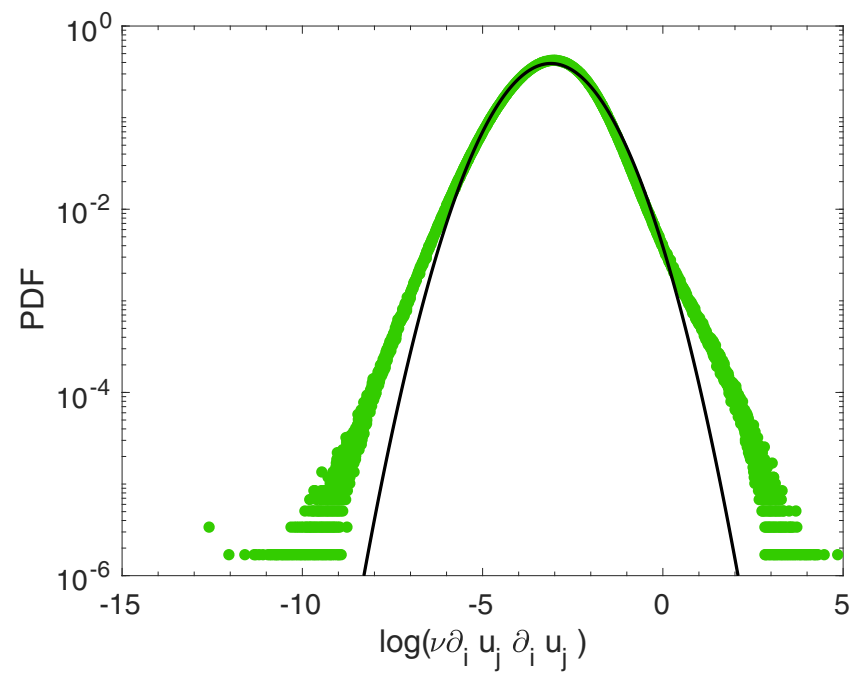

FIG. 3. Plot of the probability distribution function of the instantaneous values of $\log \left(\epsilon_{v}=v \partial_{i} u_{j} \partial_{i} u_{j}\right)$ for the experimental data set E. The black curve indicates a Gaussian with the mean and variance obtained from the experimental data set. 

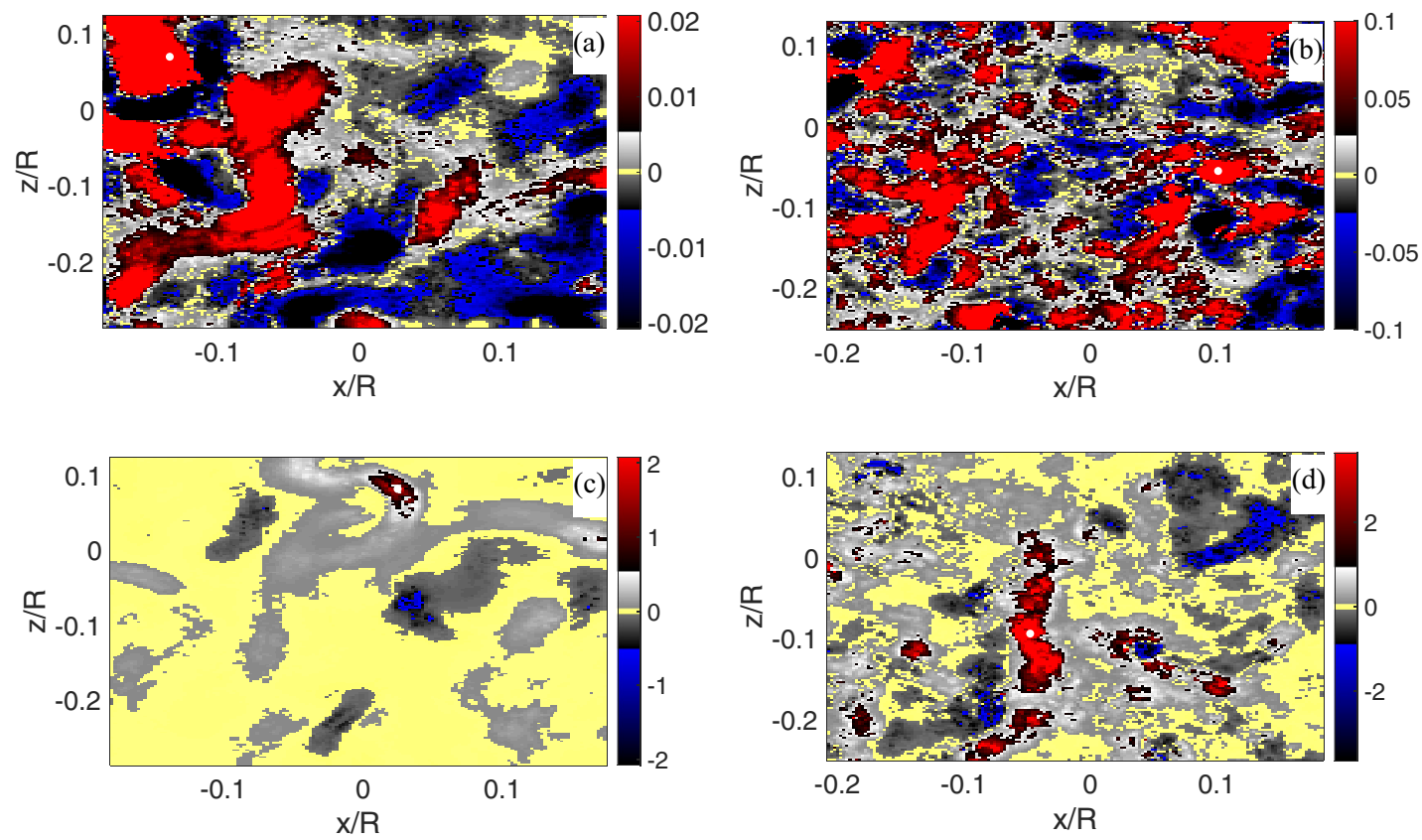

FIG. 4. Pseudocolor plots of the instantaneous $\mathscr{D}_{\ell}^{\mathrm{I}}$ field in the plane of measurement from the experimental data sets $\mathrm{E}$ and $\mathrm{C}$ at the scales $\ell=\eta$ and $\ell=12 \eta$, respectively. Top panels (a) data set $\mathrm{E}$ and (b) data set C: extreme events are absent and the location of the maximal local dissipation is shown by a white dot. Bottom panels (c) data set $\mathrm{E}$ and (d) data set $\mathrm{C}$ : in the presence of the extreme events, the white dot indicates the location of an extreme event, where the local energy transfer is 100 times larger than the mean. The measurement domain is shown in units of the radius of the cylindrical tank $R$.

$\mathscr{D}_{\ell}^{\mathrm{I}}$ in the dissipation range can be as intermittent as it is in the inertial range.

A quantitative measure of the above effect is given by the number of "extreme events" of interscale transfer, i.e., instances when $\mathscr{D}_{\ell}^{\mathrm{I}} /\left\langle\mathscr{D}_{\ell}^{\mathrm{I}}\right\rangle \geqslant 1000$ over the measurement area. In our analysis, we count the number of such events in one data set of $3 \times 10^{4}$ snapshots. We do not find any extreme event satisfying the above criterion at scale $\ell \approx 160 \eta$; only 4 extreme events at scale $\ell \approx 80 \eta ; 18$ extreme events at scale $\ell \approx 40 \eta$; and 36 extreme events at scale $\ell \approx 2 \eta$. In Fig. 4 we show the pseudocolor plots of the instantaneous $\mathscr{D}_{\ell}^{\mathrm{I}}$ for the data sets $\mathrm{E}$ and $\mathrm{C}$ at scales $\ell \approx \eta$ and $\ell \approx 12 \eta$, respectively. In particular, we show these typical maps of local energy transfer in the absence and presence of the extreme events in the upper and lower panels, respectively.

The characterization of the flow topology around the extreme events of inertial dissipation is an important question and it has been suggested that it differs from that associated with the viscous dissipation [16]. The pseudocolor plots of $\mathscr{D}_{\ell}^{\mathrm{I}}$ Figs. 4(c) and 4(d) show that these extreme events are located within the coherent structures. Moreover, another important feature which is evident from Fig. 4(c) is the presence of these extreme events of energy transfers at the scales of the order of the dissipation length scale. As a result, the local energy balance has a significant contribution from the interscale transfer, irrespective of how it is dissipated at small scales (viscous dissipation or lack of smoothness of the velocity fields $[16,22])$. This behavior is compatible with the multifractal description of turbulence [10], in which the singularities with exponent $h<1 / 3$ dissipate energy at scales $\eta_{h} \sim \operatorname{Re}^{-1 /(1+h)}<\eta$. Even though the number of extreme events increases with decreasing scale, their contribution to the total energy dissipation decreases with it. This is clear from the comparison of Fig. 2(a) and its inset. For example, if we look for events which are larger than 500 times the average dissipation rate $\epsilon$, we find only 3 such events at $\ell \sim \eta$ as compared to 306 events at $\ell \sim 12 \eta$.

The above discussion highlights the need to identify the correlation between $\mathscr{D}_{\ell}^{\mathrm{I}}$ and $\mathscr{D}_{\ell}^{v}$. We do so by computing the logarithm of the joint PDFs of these two quantities at different scales; we show them in Fig. 5. The logarithm of the joint PDFs is such that the maxima of $\left|\mathscr{D}_{\ell}^{\mathrm{I}}\right|$ coincide with minima of $\left|\mathscr{D}_{\ell}^{v}\right|$ (and vice versa). This suggests that the extreme events of interscale transfer correspond to the minima of viscous dissipation (and vice versa).

\section{Behavior of the spatiotemporal averages}

The mean of $\mathscr{D}_{\ell}^{\mathrm{I}}$ converges very slowly, if at all, perhaps because of the presence of the extreme events of both positive and negative signs in its distribution. Also, the convergence is more difficult at the large length scales than at the small length scales of a given data set; this is so because any statistical analysis of the former involves a lower number of independent blocks of data compared to the latter. In Appendix A 5 we give the details of the convergence tests, which we have performed on the following quantities, namely, $\mathscr{D}_{\ell}^{\mathrm{I}}, \mathscr{D}_{\ell}^{v}$, and $\left|\mathscr{D}_{\ell}^{\mathrm{I}}\right|$ at different scales (see Figs. 19, 20, and 21, respectively). In summary, the mean is in general not converged for any of the scales we considered for the data sets $\mathrm{B}, \mathrm{C}$, and $\mathrm{D}$, even over $3 \times 10^{4}$ frames. We observe a satisfactory convergence of the mean only for the first few scales for the data sets C and E. However, if we consider the absolute values of $\mathscr{D}_{\ell}^{\mathrm{I}}$, this 

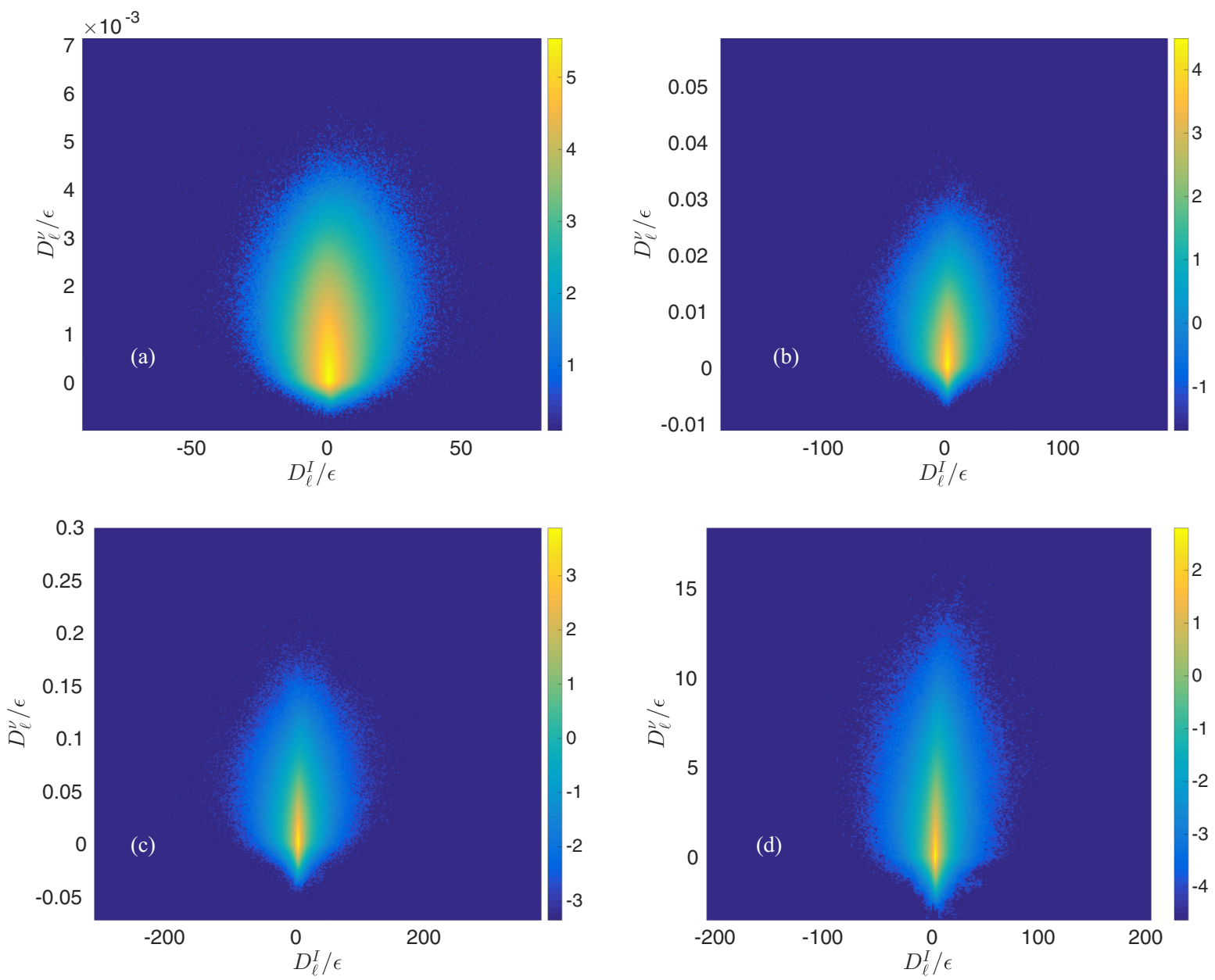

FIG. 5. Plots of the logarithm of the joint probability distribution function of the local energy transfer $\mathscr{D}_{\ell}^{\mathrm{I}}$ and the viscous dissipation $\mathscr{D}_{\ell}^{v}$ at different scales: (a) $\ell / \eta=540$, (b) $\ell / \eta=250$, (c) $\ell / \eta=70$, and (d) $\ell / \eta=3 . \eta$ is the Kolmogorov dissipation length scale and $\epsilon$ is the dimensionless energy injection rate.

problem is absent and the convergence of the mean is ensured at all scales for all the data sets A-E (over $3 \times 10^{4}$ frames).

In Figs. 6(a) and 6(b) we plot the spatiotemporal average of $\mathscr{D}_{\ell}^{\mathrm{I}} / \epsilon$ and $\left|\mathscr{D}_{\ell}^{\mathrm{I}}\right| / \epsilon$ versus universal coordinates $\ell / \eta$, respectively, for the different experimental data sets (we use different symbols to represent them). We also show the variation of the mean energy dissipation $\left\langle\mathscr{D}_{\ell}^{v}\right\rangle$ with scale $\ell / \eta$ on these plots. We remark that $\left\langle\mathscr{D}_{\ell}^{v}\right\rangle$ is second order in velocity increments and is also everywhere positive; therefore, it does not suffer from the convergence issues (see Fig. 20). Moreover, if we plot $\left\langle\mathscr{D}_{\ell}^{v}\right\rangle$ vs $\ell / \eta$, its values from different data sets collapse on a single curve. It displays $\ell^{-4 / 3}$ power-law scaling down to $30 \eta$ and it tends to $\epsilon$ at small scales. We observe that compared to $\left\langle\mathscr{D}_{\ell}^{v}\right\rangle$ the data collapse is not so good for $\left\langle\mathscr{D}_{\ell}^{\mathrm{I}}\right\rangle$ and results in scatter, a signature of the lack of convergence of this quantity for some data sets.

The behavior of absolute value of the local energy transfer $\left\langle\left|\mathscr{D}_{\ell}^{\mathrm{I}}\right|\right\rangle$ is significantly different from that of $\left\langle\mathscr{D}_{\ell}^{\mathrm{I}}\right\rangle$. The former reaches a value of about 2 to 3 times $\epsilon$ for scales $\ell>30 \eta$, whereas the latter stays below $\epsilon / 3$. This large difference can attributed to the fact that our measurements are on a plane, which prevents us from computing the contribution of the velocity increments in the azimuthal direction. This may change the proportion of the events with positive signs compared to that with negative signs, especially the extreme ones.

To check the above arguments, we compare our results with the data from the homogeneous, isotropic 3D DNS. For the DNS run we expect the sum of $\left\langle\mathscr{D}_{\ell}^{\mathrm{I}}\right\rangle$ and $\left\langle\mathscr{D}_{\ell}^{v}\right\rangle$ to be equal to the mean energy dissipation $\epsilon$. Figure 7 shows that such a relation is very well satisfied for the scales $\ell<40 \eta$, beyond which the finite-size effects lead to deviation. As expected, the relation is not satisfied for $\left\langle\left|\mathscr{D}_{\ell}^{\mathrm{I}}\right|\right\rangle$, which makes the sum exceed $\epsilon$, as now both positive and negative instances contribute without cancellations. From these plots, we can infer that the inertial range exists, if at all, for scales larger than, but close to, $10 \eta$. We observe that $\left\langle\mathscr{D}_{\ell}^{v}\right\rangle$ obtained from the DNS agrees well with the experimental data, whereas $\left\langle\mathscr{D}_{\ell}^{\mathrm{I}}\right\rangle$ obtained from the DNS is 3 times larger than that from the experiments. This observation is in agreement with the arguments about the projection effects related with SPIV, which highlights the limitation of the experimental measurements.

\section{Higher order statistics and refined similarity hypothesis}

We explore the higher order statistics of the local energy transfer and viscous dissipation by defining the following 

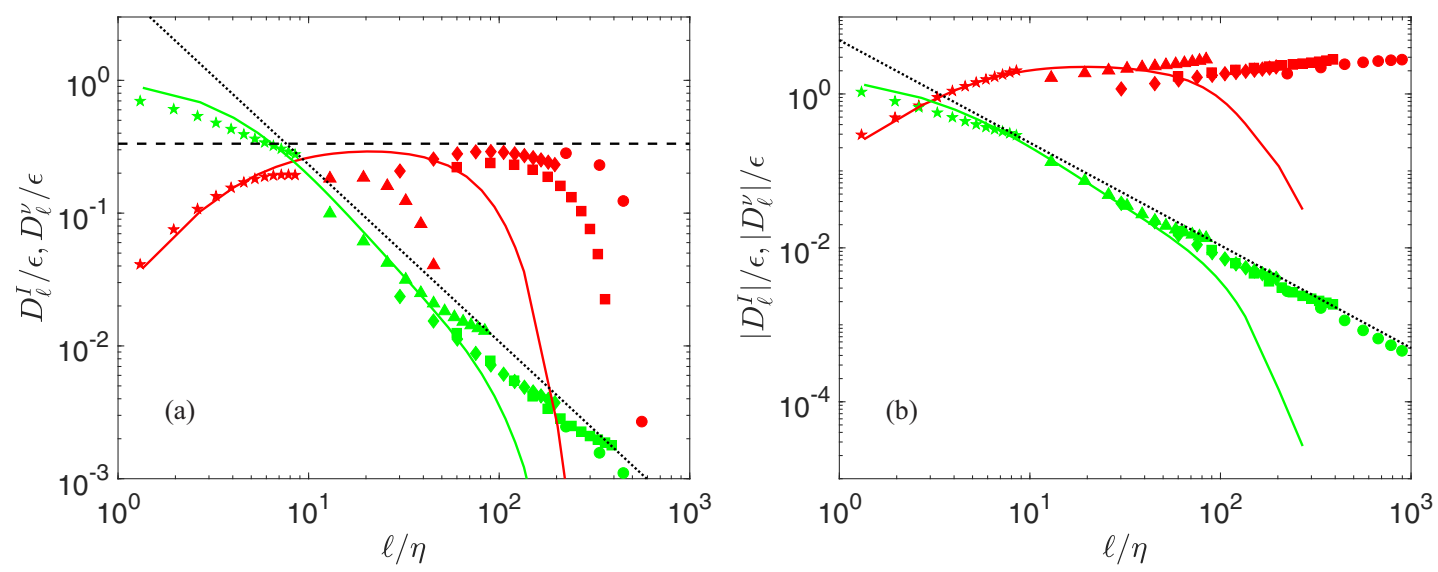

FIG. 6. Spatiotemporal averages of the local energy transfer and viscous dissipation: (a) plots of $\left\langle\mathscr{D}_{\ell}^{\mathrm{I}}\right\rangle / \epsilon$ (red symbols) and $\left\langle\mathscr{D}_{\ell}^{v}\right\rangle / \epsilon($ green symbols) vs $\ell / \eta$. (b) Analogous plots for the absolute values of the local energy transfer and the viscous dissipation. The black horizontal dashed line indicates a constant value of $1 / 3$. The black dotted line indicates the power law $\ell^{-4 / 3}$. A comparison with DNS is shown by plotting in (a) $\frac{1}{3}\left\langle\mathscr{D}_{\ell}^{\mathrm{I}}\right\rangle / \epsilon$ (red curve) and $\left\langle\mathscr{D}_{\ell}^{v}\right\rangle / \epsilon$ (green curve) vs $\ell / \eta$; (b) $2\left\langle\left|\mathscr{D}_{\ell}^{\mathrm{I}}\right|\right\rangle / \epsilon$ (red curve) and $\left\langle\left|\mathscr{D}_{\ell}^{v}\right|\right\rangle / \epsilon$ (green curve) vs $\ell / \eta$. $\eta$ is the Kolmogorov dissipation length scale and $\epsilon$ is the dimensionless energy injection rate. The different experimental data sets are represented by different symbols: A (circles), B (squares), C (diamonds), D (triangles), and E (stars).

structure functions:

$$
\begin{aligned}
& \Sigma_{p}^{\mathrm{I}}=\left\langle\left|\mathscr{D}_{\ell}^{\mathrm{I}}\right|^{p}\right\rangle, \\
& \Sigma_{p}^{v}=\left\langle\left|\mathscr{D}_{\ell}^{v}\right|^{p}\right\rangle,
\end{aligned}
$$

respectively. We compute these structure functions for $p=$ $i / 3$, where $i \in(1,9)$, and plot them in Fig. 8. We observe that for a given order $p, \Sigma_{p}^{\mathrm{I}}$ and $\Sigma_{p}^{v}$ from different data sets rescaled by $\left(\Sigma_{1}^{\mathrm{I}}\right)^{p} \eta^{\tau(p)}$ and $\left(\Sigma_{1}^{\nu}\right)^{p} \eta^{\gamma(p)}$, respectively, collapse on a single universal curve. For a given order $p$, the universal curve is a power law with exponent $\tau(p / 3)=\zeta(p)-p / 3$ and $\gamma(p / 3)$ for $\Sigma_{p / 3}^{\mathrm{I}}$ and $\Sigma_{p / 3}^{v}$, respectively. The computation of

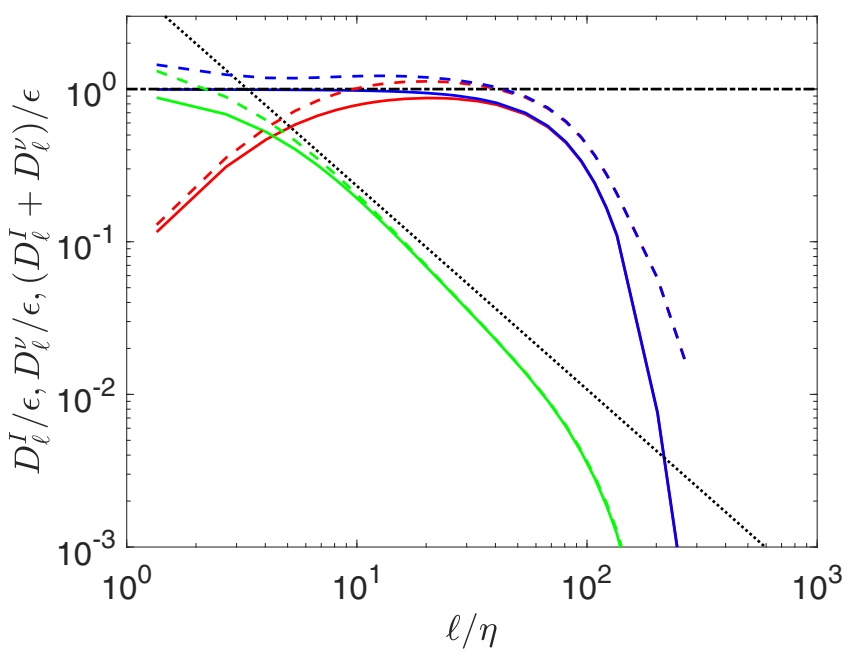

FIG. 7. Spatiotemporal averages of the local energy transfer and viscous dissipation from the DNS: plots of $\left\langle\mathscr{D}_{\ell}^{\mathrm{I}}\right\rangle / \epsilon$ (red curve) and $\left\langle\mathscr{D}_{\ell}^{v}\right\rangle / \epsilon$ (green curve) vs $\ell / \eta$. Analogous plots for the average of the absolute values of local energy transfer and the viscous dissipation are shown by dashed red and green curves, respectively. The black horizontal dashed line indicates a constant value of 1 . The black dotted line indicates the power law $\ell^{-4 / 3} . \eta$ is the Kolmogorov dissipation length scale and $\epsilon$ is the dimensionless energy injection rate. the exponent $\gamma(p / 3)$ from the multifractal theory is postponed to Sec. IIIF. We find that the magnitude of $\gamma(p / 3)$ is always smaller than the exponent $\tau(p / 3)$, which we obtain for the intermittency correction to the structure functions.

These results are in agreement with a refined similarity hypothesis, that had been suggested in [23]:

$$
\frac{S_{p}(\ell)}{\left(S_{3}(\ell)\right)^{p / 3}} \sim \frac{\Sigma_{p / 3}^{I}}{\left(\Sigma_{1}^{I}\right)^{p / 3}} .
$$

We compute these structure functions for the data from the DNS run and plot them in Fig. 8. $\Sigma_{p}^{\mathrm{I}}$ and $\Sigma_{p}^{v}$ from the DNS are in agreement with the experimental data sets for orders up to $p=4 / 3$, but show increasing deviations both at small and large length scales. We find that the agreement is better for $\Sigma_{p}^{v}$ than it is for $\Sigma_{p}^{\mathrm{I}}$, which may be attributed to the aforementioned projection effects related to SPIV.

\section{Conditioned wavelet structure functions:}

\section{The role of extreme events of inertial dissipation}

The discussion in the previous section allows us to draw an important conclusion that in the turbulent regime the intermittency corrections are governed by the local energy transfers. This motivates us to explore this connection further by conditioning the structure functions on the regions of high or low inertial dissipation (i.e., local energy transfers at the resolution scale). We implement this for each of the data sets $\mathrm{A}$ to $\mathrm{E}$ by defining two special sets of points $\mathcal{A}$ and $\mathcal{B}$ corresponding to the locations of low and high values of $\left|\mathscr{D}_{\Delta \mathrm{x}}^{\mathrm{I}}\right|$, respectively. We divide the PDF of $\left|\mathscr{D}_{\Delta \mathrm{x}}^{\mathrm{I}}\right|$ at the smallest scale of a given experimental data set (A to $\mathrm{E}$ ) into 10 deciles: the set of points forming the first decile (the spatial regions with $\left|\mathscr{D}_{\Delta \mathrm{x}}^{\mathrm{I}}\right|$ being in the $10 \%$ lower values of the inertial dissipation, that is, values close to 0 ) are assigned to the set $\mathcal{A}$ and the points forming the last decile (the spatial regions with $\left|\mathscr{D}_{\Delta \mathrm{x}}^{\mathrm{I}}\right|$ in the highest $10 \%$ in magnitude) are assigned to the set $\mathcal{B}$. Therefore, the extreme events of $\mathscr{D}_{\Delta \mathrm{x}}^{\mathrm{I}}$ are in set $\mathcal{B}$. We then 

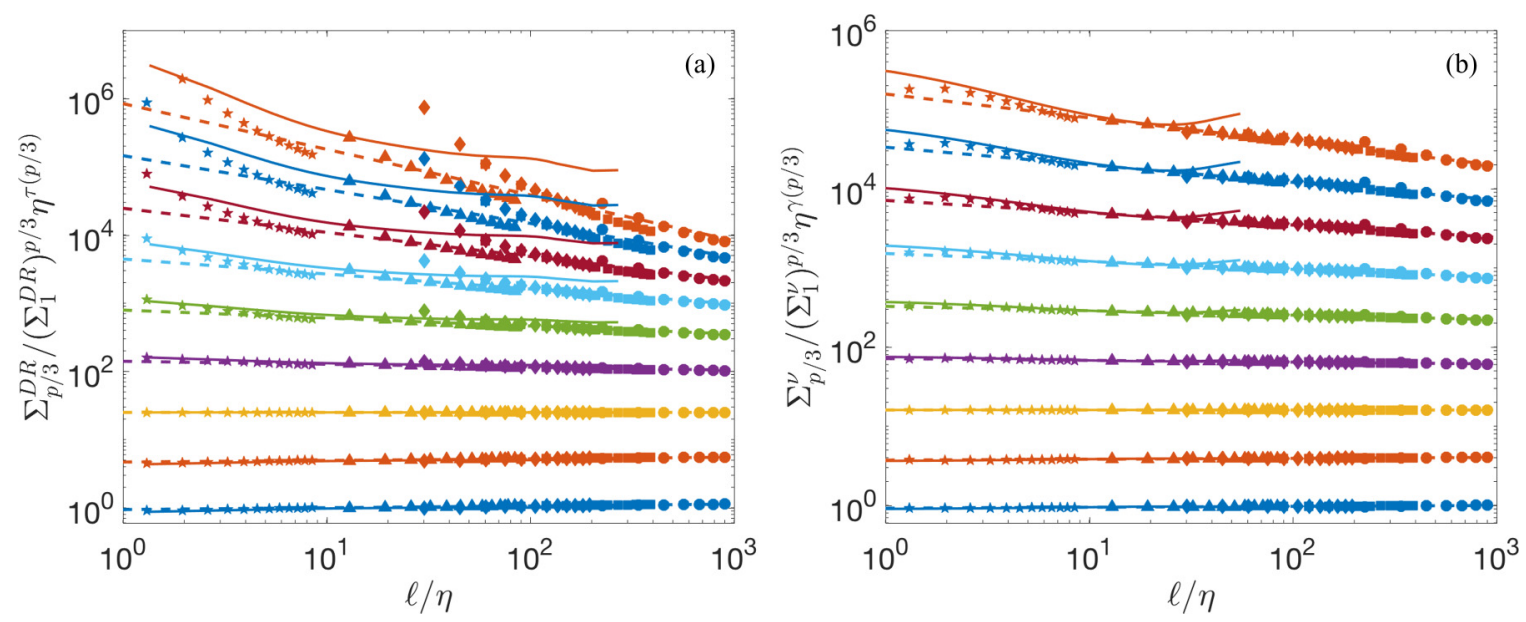

FIG. 8. Plots of the structure function of the (a) local energy transfer $\Sigma_{p}^{\mathrm{I}}$ vs $\ell / \eta$ and (b) viscous dissipation $\Sigma_{p}^{v}$ vs $\ell / \eta$. Different orders of the structure functions are distinguished by using different colors: $p=1 / 3$ (light blue), $p=2 / 3$ (orange), $p=1$ (yellow), $p=4 / 3$ (purple), $p=5 / 3$ (green), $p=2$ (pale blue), $p=7 / 3$ (red), $p=8 / 3$ (strong blue), and $p=3$ (deep orange). For the structure function of a given order, the DNS result is represented by a continuous line, while different experimental data sets are represented by different symbols: A (circles), B (squares), C (diamonds), D (triangles), and E (stars). The dotted lines indicate the power law $\ell^{\tau(p / 3)}$ (for $\Sigma_{p}^{\mathrm{I}}$ ) and $\ell^{\gamma(p / 3)}$ (for $\left.\Sigma_{p}^{\nu}\right)$; see Table II for the values of $\tau(p / 3)$ and $\gamma(p / 3)$. Note: The structure functions shown in (a) and (b) have been rescaled by $\left(\Sigma_{1}^{D R}\right)^{p / 3} \eta^{\tau(p / 3)}$ and $\left(\Sigma_{1}^{v}\right)^{p / 3} \eta^{\gamma(p / 3)}$, respectively; $\eta$ is the Kolmogorov dissipation scale.

compute the wavelet structure functions defined by

$$
S_{n}^{C}(\ell)=\left\langle|\delta W(\boldsymbol{u})(\boldsymbol{x}, \ell)|^{p}\right\rangle_{\mathcal{A}, \mathcal{B}},
$$

where the average is taken on either set $\mathcal{A}$ or set $\mathcal{B}$. We show these structure functions in Fig. 9 for orders $p \in(1,9)$ and compare them with the original, unconditioned, wavelet-based structure functions. We observe that the structure functions conditioned on the events of small inertial dissipation are less intermittent than the unconditioned ones and display $\ell^{p / 3}$ power-law scaling behavior. In contrast, the structure functions conditioned on the extreme values of the inertial dissipation have intermittency, which is similar to those that are unconditioned. This clearly shows that the intermittency is essentially governed by the extreme events of the inertial dissipation.

\section{E. Extreme value analysis of the spatiotemporal probability distribution functions}

We now know from the detailed study of the various structure functions presented in the previous sections that the extreme events of $\mathscr{D}_{\ell}^{\mathrm{I}}$ control the intermittency and their PDFs are strongly non-Gaussian. Also, these extreme events are located in the tails of the PDFs. Therefore, we now use the tools of the extreme value analysis to further explore the statistical properties of these extreme events. We characterize the tails of the PDFs by using the generalized Pareto distribution (GPD) given by

$$
f_{G P D}(x ; \mu, \sigma, \xi)=\frac{1}{\sigma}\left[1+\xi \frac{x-\mu}{\sigma}\right]^{-1-1 / \xi},
$$

where $\mu \in \mathbb{R}$ is the location parameter representing the chosen threshold, $\sigma>0$ is the scale parameter representing the typical order of extreme fluctuations, and $\xi$ is the tail shape parameter, representing the rate of decay of extremes in the tail.
We apply this analysis to the PDFs of $\mathscr{D}_{\ell}^{\mathrm{I}}$ at different scales (based on $3 \times 10^{4}$ samples) and obtain fits by using the maximum likelihood estimation criterion (implemented in the MATLAB routine "gpfit"). We fit in each case the parameters $\mu, \sigma$, and $\xi$ for the quantiles $0.975,0.99$, and 0.995 indicating strong, rare, and extreme events, respectively. As a result, we obtain the spatial and scale dependence of these three parameters $\mu(\ell), \sigma(\ell)$, and $\xi(\ell)$ for the above-mentioned three types of events. We varied the series size and find that these indicators have better convergence properties than the moments of $\mathscr{D}_{\ell}^{\mathrm{I}}$; thus, they are better suited for interpreting the behavior of the tails.

In our use of the GPD analysis, we are also motivated by the possible links it can provide with the multifractal properties of the local energy transfer field $\mathscr{D}_{\ell}^{\mathrm{I}}(\boldsymbol{x}, t)$. The GPD analysis of a multifractal field, characterized by a spectrum $D(h)$, yields the following scale dependence for the three parameters:

$$
\mu(\ell) \sim \ell^{h_{\min }}, \quad \sigma(\ell) \sim \ell^{h_{\min }}, \quad \xi \sim 1 / p_{*},
$$

where $h_{\min }=\min [h / D(h)=0]$ and $p_{*}=\left.\frac{d D(h)}{d h}\right|_{h_{\min }}$ (see Refs. [24,25] for additional details).

\section{Quantile value parameter $\mu$}

The parameter $\mu$ describes the value at which a given quantile is achieved, i.e., the typical value of an extreme event that has the probability given by the quantile. In Figs. 10(a) and 10(b) we show the variation of $\mu$ normalized by $\epsilon$ as a function of $\ell / \eta$ from the negative and positive tails of the PDF, respectively. We observe that for the positive tails [see Fig. 10(b)], the normalized quantile threshold $\mu / \epsilon$ follows a smooth curve, which is almost flat over the range of scales $(10 \eta, 300 \eta)$ coinciding with the inertial range. This behavior corresponds to the value $h_{\text {min }}=0$. We also find that outside the above-mentioned range of scales, both at the small and large length scales, $\mu$ decreases to zero rather sharply. For the 

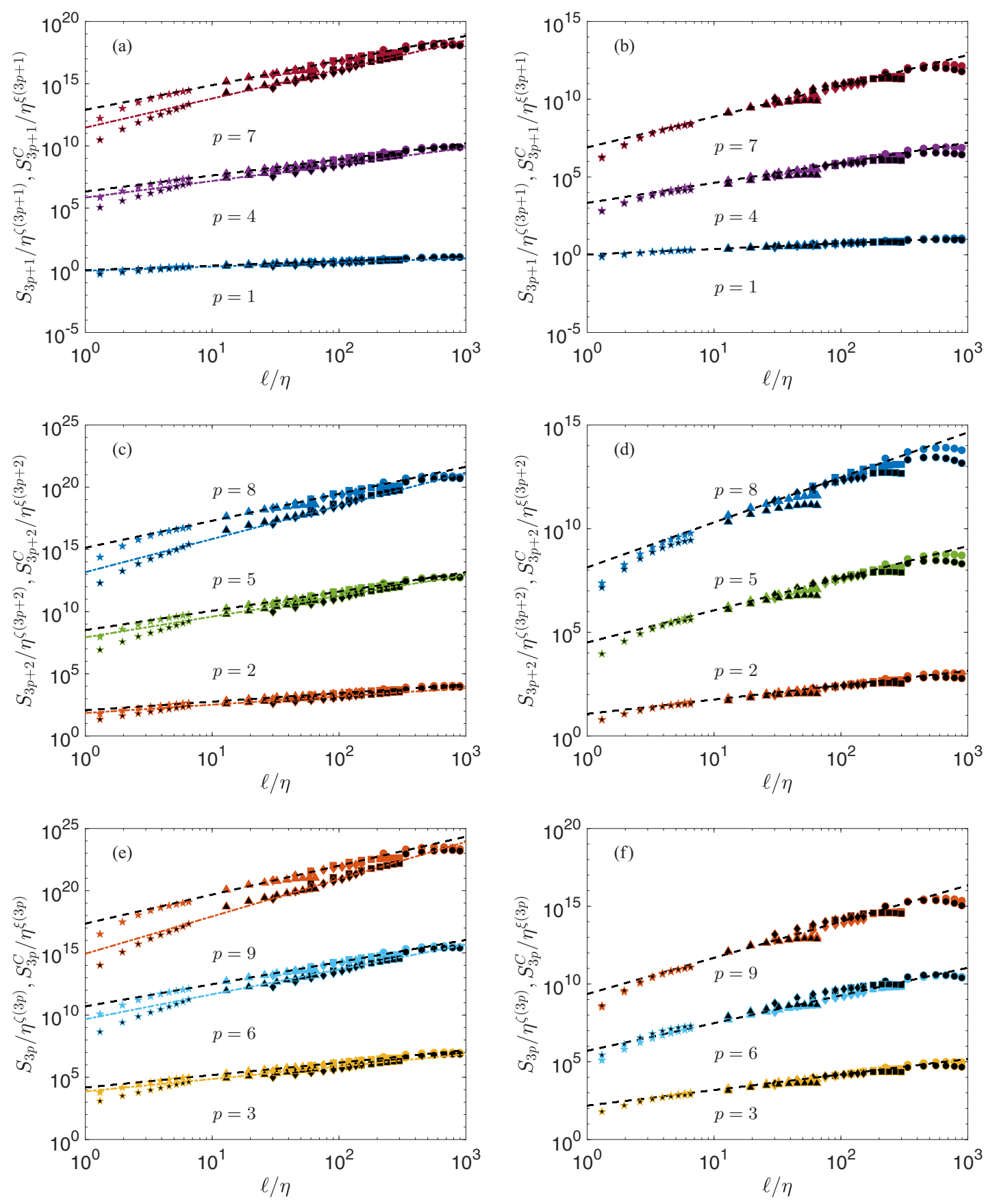

FIG. 9. Role of the extreme events of inertial dissipation. Plots of the structure functions: $S_{p}^{C}$ vs $\ell / \eta$ conditioned (symbols with black filling) either on the set $\mathcal{A}$ (less intense events of $\mathscr{D}_{\Delta}^{\mathrm{I}} x$; shown in the left panel) or $\mathcal{B}$ (extreme events of $\mathscr{D}_{\Delta}^{\mathrm{I}} x$, shown in the right panel) and the unconditioned $S_{p}$ vs $\ell / \eta$. The black dashed lines indicate $\ell^{\zeta(n)}$. The colored lines show $\ell^{p / 3}: p=1$ (light blue), $p=2$ (orange), $p=3$ (yellow), $p=4$ (purple), $p=5$ (green), $p=6$ (pale blue), $p=7$ (red), $p=8$ (strong blue), and $p=9$ (deep orange). The structure functions have been shifted by multiplying with an arbitrary factor for visual clarity.

negative tails [see Fig. 10(a)], the plots of $\mu$ do not follow a smooth curve and are more scattered. This difference between the behavior of the positive and negative tails may be an indication of the fact that the positive and negative events of $\mathscr{D}_{\ell}^{\mathrm{I}}(\boldsymbol{x}, t)$ are not equivalent and correspond to different processes. Moreover, we notice discontinuities on moving from one experimental data set to another, thereby implying that $\mu$ is sensitive to the Reynolds number. In Fig. 10(c) we show the plots of $\mu_{-} / \epsilon$ versus $\mu_{+} / \epsilon$ and we find that on average the $\mu_{+}$is larger than $\mu_{-}$, tracing the asymmetry of the distribution towards positive values.

\section{Intermittency parameter $\sigma$}

The parameter $\sigma$ quantifies the "width" of the tails of a PDF; therefore, it is an indicator of the importance of the extreme events. In Figs. 11(a) and 11(b) we plot the $\sigma$ normalized by $\epsilon$ versus $\ell / \eta$ for the negative and the positive tails of the PDFs, 

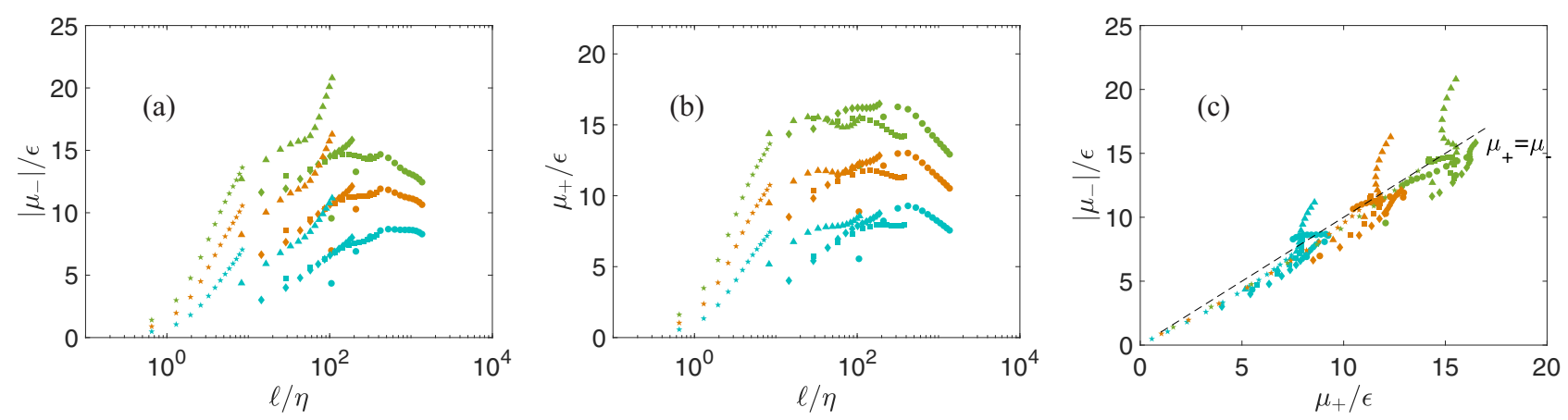

FIG. 10. Quantile threshold parameter from the GPD analysis: (a) $\mu_{-} / \epsilon$ vs $\ell / \eta$ and (b) $\mu_{+} / \epsilon$ vs $\ell / \eta$ from the negative and positive tails of the PDF of $\mathscr{D}_{\ell}^{\mathrm{I}}$, respectively. (c) Parametric plot of $\mu_{-} / \epsilon$ vs $\mu_{+} / \epsilon$. Different colors indicate different quantile values: 0.975 (cyan), 0.99 (orange), and 0.995 (green). The dotted line indicates $\left|\mu_{+}\right|=\left|\mu_{-}\right|$. Different experimental data sets are indicated by different symbols: A (circles), B (squares), C (diamonds), D (triangles), and $\mathrm{E}$ (stars). $\eta$ is the Kolmogorov length scale.

respectively. We observe that, when plotted in this manner, only the lowest or the lower two quantiles follow a universal curve for the different experimental data sets $\mathrm{A}$ to $\mathrm{E}$ and this behavior is independent of whether we choose the positive or the negative tail. For the highest quantile, we notice a Reynolds number dependence in the form of sharp discontinuities on moving from one data set to the next. We may attribute the observation of this behavior to the lack of statistics-the higher the quantile the lower the number of events-therefore, resulting in a poor fit. However, at the highest quantile, we can improve the data collapse of different experimental data sets by explicitly introducing the Reynolds number dependence. This is based on the assumption that the intermittency parameter follows a shape governed by the similarity of second kind, first suggested by Castaing [26,27] and derived by Dubrulle and Graner from finite scale invariance [28]:

$$
\ln \sigma=\beta(\exp [\alpha \ln (\ell / L)]-1),
$$

where $\beta=\beta_{0} \ln (\operatorname{Re})$ and $\alpha=\alpha_{0} / \ln (\operatorname{Re})$, and $L$ is a large scale ( $L=1$ in our nondimensional units). In the limit $\ln (\mathrm{Re}) \rightarrow 0$, we recover the classical similarity $\ln (\sigma) \propto \ln (\ell / L)$, but at finite $\operatorname{Re}$ we have to include corrections to the similarity. In the present case, this assumption implies that $\ln (\sigma) / \ln (\operatorname{Re})$ versus $\ln (\ell) / \ln (\operatorname{Re})$ should collapse on a universal curve for the different data sets. We show this in the insets Figs. 11(a.1) and 11(b.1) for the negative and the positive tails of the PDFs, respectively. As we expected, the collapse between the different data sets is better for the highest quantile. Moreover, the curve is a straight line, as required by the shape relation Eq. (23). However, the slope of the straight line, which traces $h_{\text {min }}$, depends on the quantile: it increases from 0 to -0.25 as the quantile changes from 0.975 to 0.995 , respectively. This means that $h_{\min }$ decreases with the value of the quantile. We find that in all the cases the value of $h_{\min }$ is smaller than those inferred by using the parameter $\mu$.

In Fig. 12 we show the plots of $\sigma_{-} / \epsilon$ vs $\sigma_{+} / \epsilon$, where $\sigma_{-}$ and $\sigma_{+}$denote the intermittency parameters for the negative and positive tails of the PDFs, respectively. We observe that these parametric curves display some curvature, indicating that the two quantities are not correlated in a simple way. In addition, we find that the intermittency parameter of the positive tail has the tendency to be higher than the negative tails; this implies that the positive extreme events are more intermittent than those with negative sign.
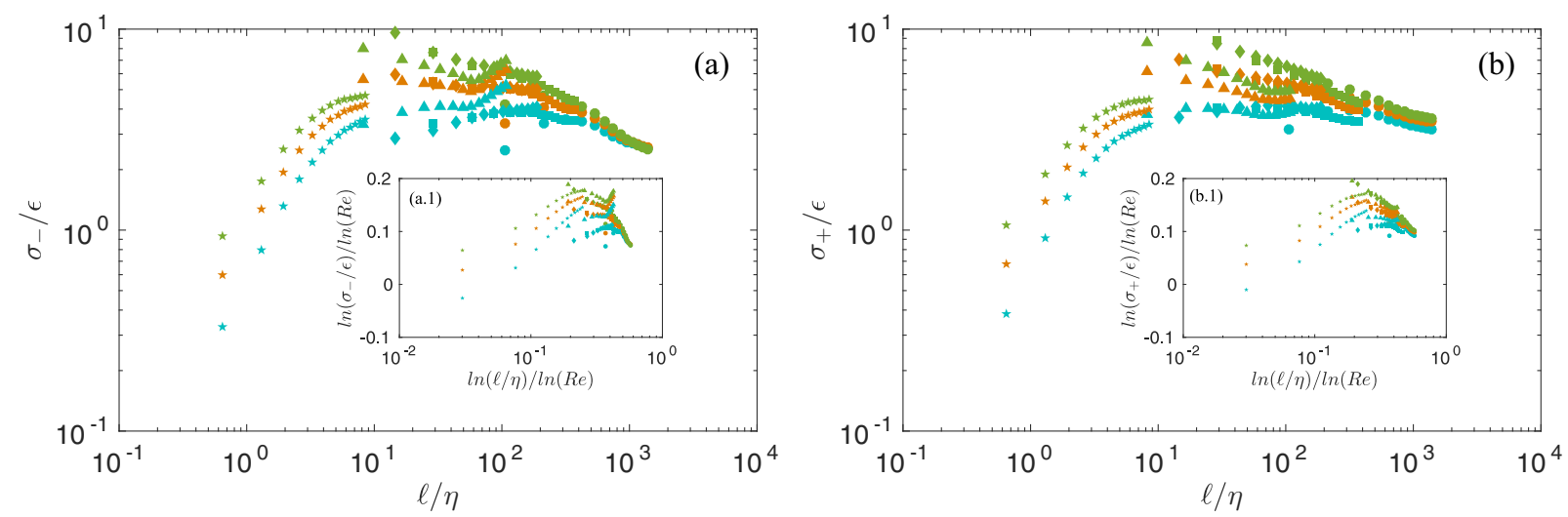

FIG. 11. Intermittency parameter from the GPD analysis: (a) $\sigma_{-} / \epsilon$ vs $\ell / \eta$ and (b) $\sigma_{+} / \epsilon$ vs $\ell / \eta$ for the negative and positive tails of the PDF of $\mathscr{D}_{\ell}^{\mathrm{I}}$, respectively. Insets: (a.1) and (b.1) show the analogous plots of $\sigma$ obtained by using the self-similarity of the second kind, i.e., plots of $\ln (\sigma / \epsilon) / \ln (\operatorname{Re})$ vs $\ln (\ell / \eta) / \ln (\operatorname{Re})$, where Re is the Reynolds number. Different colors indicate different quantile values: 0.975 (cyan), 0.99 (orange), and 0.995 (green). Different experimental data sets are indicated by different symbols: A (circles), B (squares), C (diamonds), $\mathrm{D}$ (triangles), and $\mathrm{E}$ (stars). $\eta$ is the Kolmogorov length scale. 


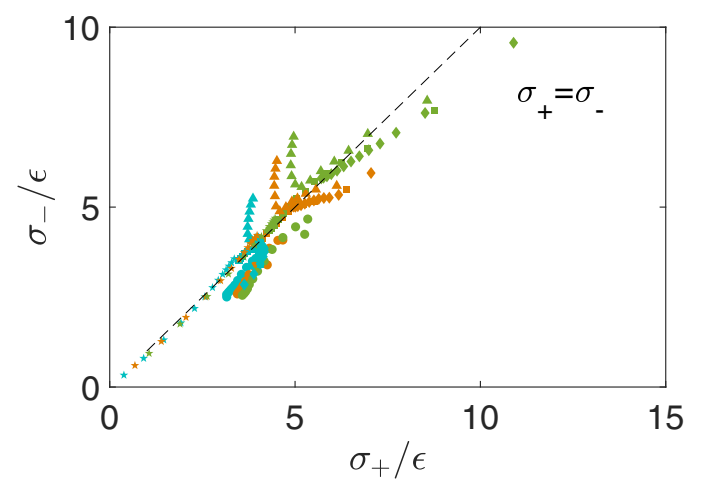

FIG. 12. Intermittency parameter: Parameteric plot of $\sigma_{-} / \epsilon$ vs $\sigma_{+} / \epsilon$. Different colors indicate different quantile values: 0.975 (cyan), 0.99 (orange), and 0.995 (green). Different experimental data sets are indicated by different symbols: A (circles), B (squares), C (diamonds), $\mathrm{D}$ (triangles), and $\mathrm{E}$ (stars). $\sigma_{-}\left(\sigma_{+}\right)$denotes the intermittency parameter for the negative (positive) tail of the PDF of $\mathscr{D}_{\ell}^{\mathrm{I}}$.

\section{Power-law parameter $\xi$}

The parameter $\xi$ quantifies the index of the power law of an equivalent GPD. If $\xi \leqslant 0$, this indicates that the distribution is bounded and well behaved. The $\xi>0$ implies a pathological distribution with diverging moments: if the shape parameter $\xi>1 / n$, the moments of order $n$ and greater do not exist [29]. In Figs. 13(a) and 13(b) we plot the parameter $\xi$ vs $\ell / \eta$ for the negative and positive tails of the PDFs, respectively; we show analogous plots in the insets Figs. 13(a.1) and 13(b.1) for this parameter vs $\ell / R$ for the negative and positive tails of the PDFs, respectively, where $R$ is the radius of the cylindrical tank. Figures 13(a.1) and 13(b.1) show that the curves for different quantiles collapse on each other indicating that the Kolmogorov length scale is not the relevant scale to explain the behavior of this parameter. $\xi$ is close to zero at large length scales and increases continuously as we decrease the scale; at the smallest scale in our data sets its value is close to 0.6 for the highest quantile. This means that the second-order moments are not defined and it also explains why it is so difficult to achieve the convergence of the mean.

The steepening of $\xi$ vs $\ell$ implies that the parameter $p_{*}=1 / \xi$ decreases as a function of scale. Moreover, at the Kolmogorov scale, we do not observe any indication of the saturation of the power-law parameter, which means that we are potentially exploring more and more singular regions.

Figure 14 shows that the correlation plot of $\xi$ from the negative tail $\left(\xi_{-}\right)$vs that from the positive tail $\left(\xi_{+}\right)$of the PDFs is roughly linear; we also observe that $\xi_{-}$has a tendency to be larger than $\xi_{+}$. This means that the convergency to a singularity, if any, is faster for the extreme events of $\mathscr{D}_{\ell}^{\mathrm{I}}$ with negative sign.

\section{F. Compatibility with multifractal analysis}

The above GPD analysis has provided us with two important pieces of information regarding the multifractal spectrum of the local energy transfer: (1) $h_{\min }$ is between -0.25 and 0 with dependence on the quantity and quantile considered; (2) $p_{*}$ changes between 1.67 and $\infty$, if we move from the Kolmogorov scale to the injection scale. We compare this prediction with the direct estimates of these quantities obtained from the scaling exponents $\tau(p)$ listed in Table II. We use the Legendre transform formula

$$
\tau(p)=\min _{h}[p h+2-D(h)]
$$

to obtain the values of $D(h)$, which we show in Fig. 15 . We obtain $h_{\min }=-0.88$ and $p_{*}=4$. This value of $h_{\min }$ is much smaller than that obtained from the GPD analysis, which suggests that the latter requires higher quantile to converge this quantity. The value of $p_{*}$ obtained by using $D(h)$ is obtained in the GPD analysis for scales close to $\ell=10^{-2} R(=1 \mathrm{~mm})$, which sets the characteristic size of the structure. We recall that the local energy transfer scales as $h=3 h_{v}-1$, where $h_{v}$ is the scaling exponent of the velocity. This suggests that the above structures correspond to those with $h_{v} \approx 0$. This estimate is compatible with the observations of Saw et al. [16] of extreme events of inertial dissipation, that look as shock- or front-like
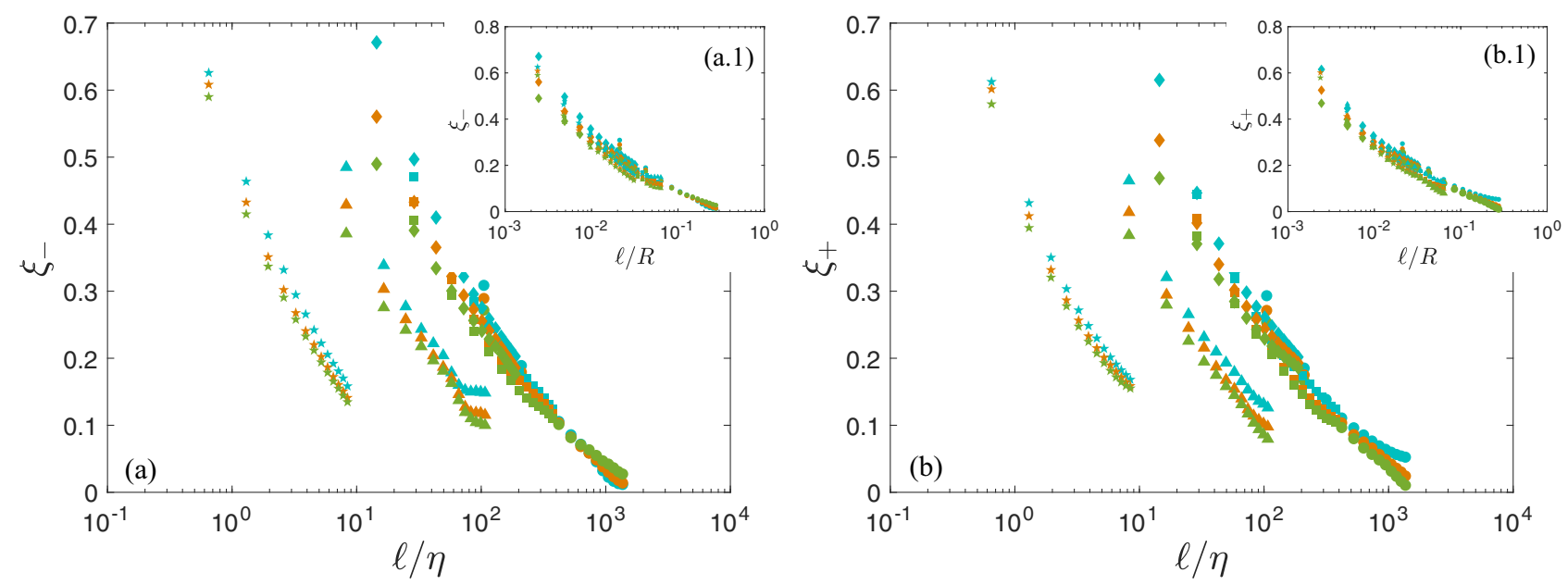

FIG. 13. Power-law parameter from the GPD analysis: (a) $\xi_{-}$vs $\ell / \eta$ and (b) $\xi_{+}$vs $\ell / \eta$ for the negative and positive tails of the PDF of $\mathscr{D}_{\ell}$, respectively. Insets: (a.1) and (b.1) show the analogous plots of $\xi$ vs $\ell / R$, where $R$ is the radius of the cylindrical tank. Different colors indicate different quantile values: 0.975 (cyan), 0.99 (orange), and 0.995 (green). Different experimental data sets are indicated by different symbols: A (circles), B (squares), C (diamonds), D (triangles), and E (stars). $\eta$ is the Kolmogorov length scale. 


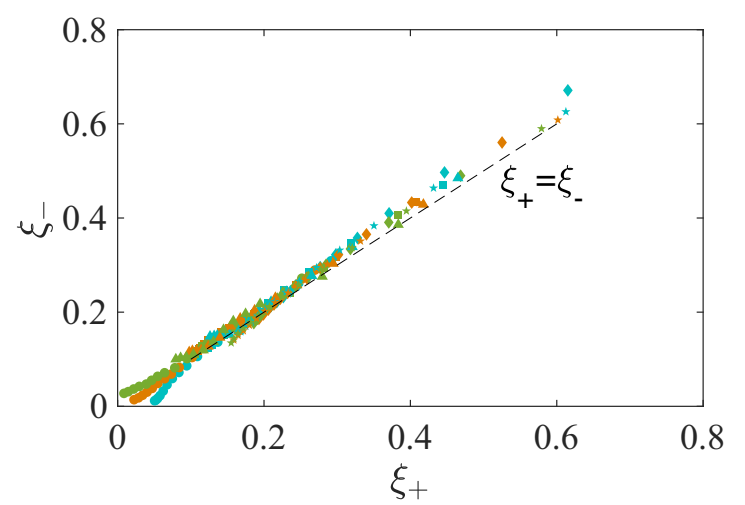

FIG. 14. Power-law parameter from the GPD analysis: Parametric plot of $\xi_{-}$vs $\xi_{+}$. Different colors indicate different quantile values: 0.975 (cyan), 0.99 (orange), and 0.995 (green). Different experimental data sets are indicated by different symbols: A (circles), B (squares), $\mathrm{C}$ (diamonds), D (triangles), and $\mathrm{E}$ (stars). $\xi_{-}\left(\xi_{+}\right)$is the power-law parameter for the negative (positive) tail of the PDF of $\mathscr{D}_{\ell}^{I}$.

structures with elongated shape, of about $10 \Delta x$ in the direction perpendicular to the front.

The obtained $D(h)$ can also be used to compute other quantities of interest, like the exponent $\gamma$. Indeed, since $\mathscr{D}_{\ell}^{v}$ scales like $2 h-2$, we get

$$
\begin{aligned}
\left\langle\left|\mathscr{D}_{\ell}^{v}\right|^{p}\right\rangle & \sim \ell^{\xi_{v}(p)}, \\
\xi_{v}(p) & =\min _{h}[p(2 h-2)+2-D(h)] .
\end{aligned}
$$

Approximating $C(h)=2-D(h)$ by a parabola $C(h)=(h-$ $a)^{2} / 2 b$, with $a=1 / 3+3 b / 2$ to ensure that $\zeta(3)=1$, we get

$$
\begin{aligned}
\xi_{v}(p) & =2 p(a-1)-2 b p^{2}, \\
\gamma(p / 3) & =\frac{2}{3} b p-\frac{2}{9} b p^{2} .
\end{aligned}
$$

Using $b=0.025$, we get the value of Table II.

\section{DISCUSSION AND CONCLUSIONS}

We know that at small scales turbulence displays strong deviations from the homogeneity and self-similarity assumptions of Kolmogorov's phenomenological theory. In this work, we have carried out a detailed exploration of the idea that these strong deviations are linked to the existence of singularities or quasisingularities in the turbulent flows, which produce extremely localized and violent events of dissipation $[14,15]$. Therefore, both the homogeneity and the regularity conditions have to be relaxed and the weak formulation approach needs to be adopted to describe the local energy transport and dissipation [11]. We discuss in detail the local energy balance equation derived by using the weak solution formulation (see Refs. [11,12]). In particular, we elaborate on the expressions for the local energy transfers and local energy dissipation; we show how in our approach they generalize the average quantities in the Kármán-Howarth-Monin equation. We call this framework the weak Kármán-Howarth-Monin (WKH) equation.

We emphasize that this approach offers several interesting possibilities, as we describe below. WKH provides a natural framework to study possible quasisingularities and

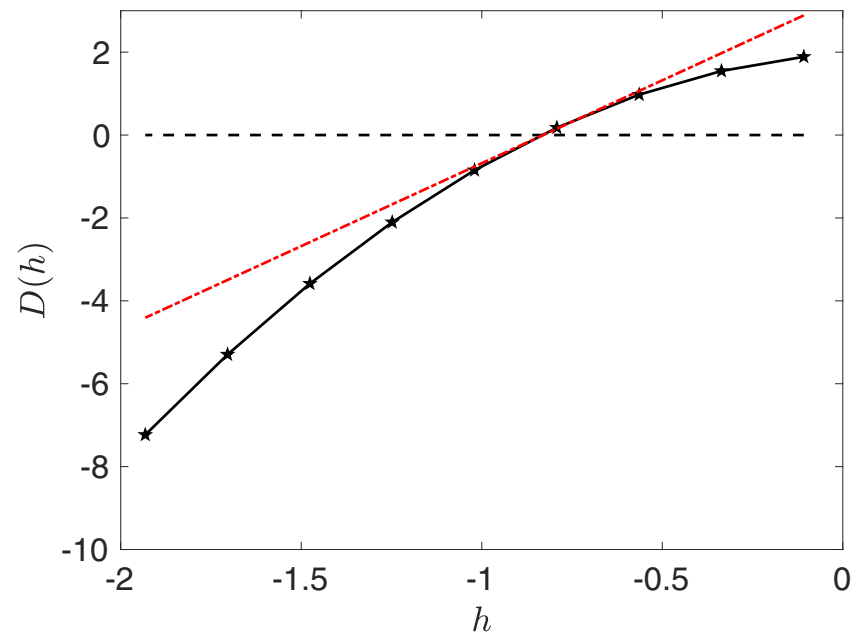

FIG. 15. Multifractal spectrum: Plot of $D(h)$ vs $h$ computed by using the values of the $\tau(p)$, scaling exponents of the local energy transfer structure functions (see Table II), in Eq. (24). The red dash-dot line indicates the tangent $f(h)=4\left(h-h_{\min }\right)$, where $h_{\min }=-0.88$.

singularities (see Ref. [22] for more details). In the presence of a quasisingularity or a singularity in the velocity field, it yields a nonzero inertial dissipation term $\mathscr{D}^{\mathrm{I}}$ in the limit $\ell \rightarrow 0$. Moreover, even after we perform a coarse-graining on the velocity field, the signatures of quasisingularities and singularities are present at finite resolutions in the form of $\mathscr{D}_{\ell}^{I}$ being nonzero along the lines in the scale space that originate from the singularity at $\ell=0$. We can use the study of the velocity field at the dissipation scales around the extreme events of $\mathscr{D}_{\ell}^{\mathrm{I}}(\boldsymbol{u}, \boldsymbol{x}, t)$ to classify the possible quasisingularities and singularities [16]. Therefore, WKH provides a concrete description of the multifractal framework. Also, the analysis of the tail of the PDFs of $\mathscr{D} \mathrm{I}(\boldsymbol{u}, \boldsymbol{x}, t)$ provides information about the topology of the quasisingularities.

The main advantage of the WKH framework is to localize in space and time the different terms, which contribute to the energy cascade at any scale $\ell$ and associate them with special topologies of the velocity fields. This approach can be then used far beyond the Kolmogorov scale, e.g., to study energy transfers in geophysical flows [30]. We find this to be complementary to the traditional methods used in turbulence, which focus only on the structures associated with enstrophy or viscous dissipation (these were thought be the only relevant entities as the end product of the energy cascade). In our framework, the local energy transfer and inertial dissipation term appears as the most relevant quantity, which may be used to devise and validate new models of turbulence. Also, we can compute the main terms describing the local energy transfers $\mathscr{D}_{\ell}^{\mathrm{I}}$ and $\mathscr{D}_{\ell}^{v}$, once the velocity field is known. Interestingly, as discussed in Ref. [22], it is now possible to explore these concepts in experiments since the advent of the particle image velocimetry methods. Moreover, the weak formulation introduces a natural smoothing that makes the computation of these quantities much less sensitive to the noise than the original direct formulation.

Motivated by these considerations, we use the experimental measurements in a turbulent swirling flow to provide a study of the statistics and the scaling properties of $\mathscr{D}_{\ell}^{\mathrm{I}}$ and $\mathscr{D}_{\ell}^{v}$. We 
find that the energy transfers are very intermittent because of the presence of extreme events at the dissipative scale, which may be regarded as the footprints of the quasisingularities or singularities existing at sub-Kolmogorov scale [16]. We show that these extreme events govern the intermittency corrections of the velocity field and follow from a refined similarity analysis based on local energy transfers [6,31]. We characterize the distribution of these extreme events by using the generalized Pareto distribution (GPD) analysis. The width of the tails is shown to be compatible with a similarity of the second kind, first proposed by Castaing [27]. Finally, we make a connection between the topological and the statistical properties of the extreme events of the inertial dissipation field and its multifractal properties.

In our analysis we are constrained to use the velocity fields measured on a plane because of the experimental limitations. We recognize that it is important to perform the same analysis on the velocity field measured over a volume, but the latter is still an experimental challenge because of the extreme refinement needed for such an analysis. We partly fill this gap by performing a 3D DNS at a moderate resolution and check that our results are not affected by the measurement constraints. Given that the numerical simulations of the INSE are performed over a finite resolution, it is yet not clear whether the extreme events of the local energy transfer will still be present, if they are indeed triggered by the potential sub-Kolmogorov scale Navier-Stokes singularities or quasisingularities. In the absence of these extreme events, we expect a different behavior of the tails of the distributions of the local energy transfers. However, an elaborate validation of our experimental results in numerical simulations requires very large simulations, which is a challenging task.

\section{ACKNOWLEDGMENTS}

This work has been supported by the Labex PALM (project Interdist), by EuHIT, a project funded by the European Community Framework Programme 7, Grant Agreement No. 312778, and by the ANR EXPLOIT, Grant Agreement No. ANR-16-CE06-0006-01. We thank the anonymous referee for very constructive remarks that helped to improve substantially the paper.

\section{APPENDIX: METHODS}

\section{Experimental setup}

We visualize turbulent von Kármán flows in a plexiglass cylinder of radius $R=100 \mathrm{~mm}$, filled with water at a controlled temperature of $20^{\circ} \mathrm{C}$. The flows are driven by two counterrotating curve-bladed impellers at a frequency $F$, rotating with their blades concave face pushing forward (called ANTI in previous publications of our group). The setup is oriented with its axis of symmetry in the vertical direction. Using high-zooming lenses coupled to standard stereo particle image velocimetry (PIV), we obtain 3-component velocity fields (radial $u_{r}$, axial $u_{z}$, azimuthal $u_{\phi}$ ) of the flows in a vertical plane containing the symmetric axis, with nominal spatial resolution between $\Delta x=0.24 \mathrm{~mm}$ and $\Delta x=3.4 \mathrm{~mm}$ (the laser sheet that defines the measurement plane is $\sim 1 \mathrm{~mm}$ thick). The torque and frequency of each impeller is measured using a

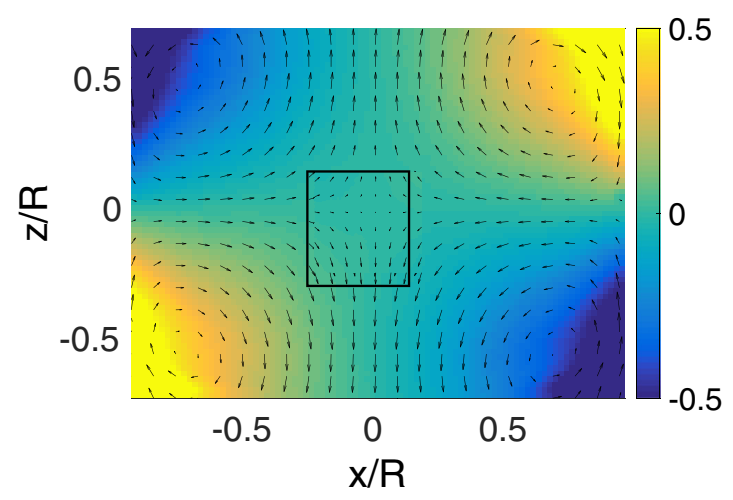

FIG. 16. Patchwork of mean velocity field in experiments A and $\mathrm{C}$ (in the black square). Color codes $u_{y}$, while $u_{x}$ and $u_{z}$ are in arrows. The coordinates have been nondimensionalized by $R$ the radius of the cylinder.

torque meter (SCAIME Inc.). More details on the setup can be found in [16]. The Reynolds number of the flow is given by $\operatorname{Re}=2 \pi R^{2} F / \nu$, where $\nu$ is the fluid's kinematic viscosity. We use different mixtures of glycerol and water in order to vary the viscosity of the working fluid, and thus the Reynolds number. Monitoring the torques $C_{1}$ and $C_{2}$ applied to each impeller, we obtain the dimensionless energy injection rate as

$$
\epsilon=\frac{2 \pi\left(C_{1}+C_{2}\right) F}{\rho \pi H R^{4}(2 \pi F)^{3}}
$$

We have checked in a scale 4:1 heat-insulated version of our experiment in helium that in the stationary regime, the global energy input is balanced by a global heat output, so that $\epsilon$ also measures the energy dissipated in the flow. Due to flow inhomogeneity, however, the energy dissipation is not homogeneous within the whole flow. Therefore, we use in this paper local estimates, derived from second-order structure functions in [17]. From this, we can compute the Kolmogorov dissipative scale as $\eta=\left(\epsilon / \nu^{3}\right)^{-1 / 4}$.

We have further shown that in our experimental setup the dimensionless energy dissipation rate saturates towards a constant value $\epsilon=0.05$ above $\operatorname{Re}_{c}=3500$ [32], corresponding to the critical Reynolds number for the onset of fully developed turbulence [33]. Varying the viscosity while keeping $\mathrm{Re}>3500$ thus enables us to monitor the size of the dissipative scale, while remaining in the regime where the flow is fully turbulent. Measurements at different values of $\Delta x / \eta$ were then obtained by acting on the frequency $F$, the mixture composition, and the zooming lens. Table I summarizes the parameters corresponding to the different cases. In cases A and B, the SPIV system has been zoomed on a $4 \mathrm{~cm} \times 3 \mathrm{~cm}$ zone at the center of the experiment; see Fig. 16.

\section{Velocity fields}

The results of this paper are based on series of SPIV measurements taken at frequency $15 \mathrm{~Hz}$ ( 3 to 12 times the eddy turnover time) over 35 to 40 minutes, resulting in samples of 30000 frames that can be considered as statistically independent. For each sample, the velocity field is reconstructed using peak correlation performed over $50 \%$ overlapping windows of size 16 to 32 pixels. As a result, we get instantaneous 

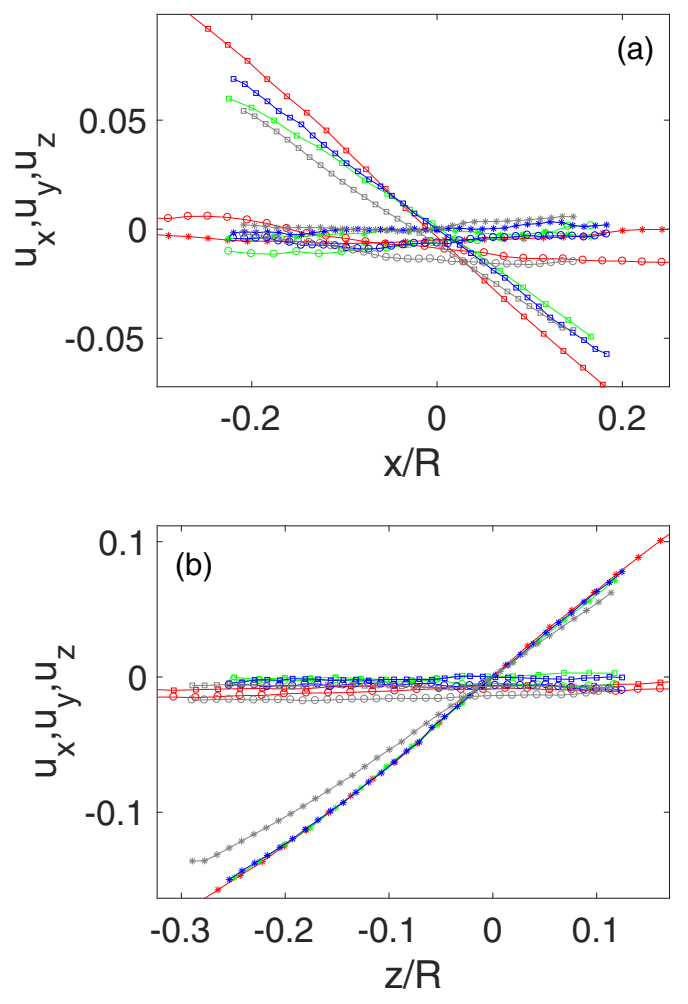

FIG. 17. Radial (a) and vertical (b) velocity profiles at $z=0$ (resp. $x=0$ ). Red: case A; green: case B; blue: case C; gray: case E. Square: $u_{r}$; circles: $u_{y}$; stars: $u_{z}$. The coordinates have been nondimensionalized by $R$ the radius of the cylinder.

snapshots of the three components of the velocity field on a grid of approximate size $90 \times 70$. In the sequel, we work with dimensionless quantities, using the radius of the vessel $R$ as the unit of length, and the impeller rotation period $(2 \pi F)^{-1}$ as the unit of time. A typical map of the time-averaged velocity fields for the global experiments is provided in Fig. 16 for the cases $\mathrm{A}$ and $\mathrm{C}$, showing the location of the zoom window. One observes a continuity in the flow topology between the zoomed and unzoomed case, showing that the calibration is consistent in between the two cases. To quantify further the discrepancy between the different zoomed and unzoomed field and estimate error bars, we plot in Fig. 17 mean radial and vertical profiles near the stagnation point. From this comparison, we can estimate a relative error on velocity measurements of $10 \%$, resulting in an uncertainty of $30 \%$ for third-order quantities.

\section{Wavelet spectrum}

Given that the weak formulation was derived using continuous wavelet transform, it is informative to first compute the wavelet power spectrum of our velocity fields, given by

$$
E^{W}(k)=\frac{1}{C_{\Psi} k_{\Psi}} \int d^{2} b\left|W T_{i}\left(\Psi, \boldsymbol{u}, \frac{k_{\Psi}}{k}, b\right)\right|^{2},
$$

where

$$
W_{i}(\Psi, \boldsymbol{u}, a, b)=\int u_{i}(x) \Psi\left(\frac{x-b}{a}\right) \frac{d^{2} x}{a}
$$

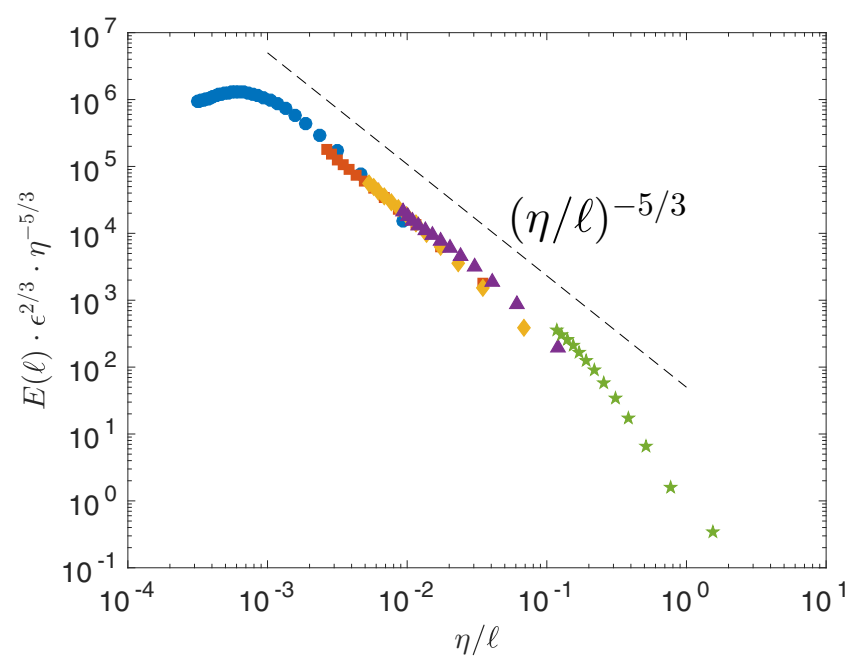

FIG. 18. Wavelet spectrum for the 5 different cases of Table I: blue circle: case A; red square: case B; yellow diamond: case C; purple triangle: case $\mathrm{D}$; green stars: case $\mathrm{E}$. The dotted line is $(\eta / \ell)^{-5 / 3}$. The spectrum and the the scale $\ell$ have been made nondimensional using $\eta$, the Kolmogorov scale, and $\epsilon$, the energy dissipation.

is the $2 \mathrm{D}$ continuous wavelet transform of $u_{i}$ with respect to the Mexican hat wavelet $\Psi$ (Laplacian of a Gaussian), $C_{\Psi}$ is a normalizing coefficient, and $k_{\Psi}$ is the centroid velocity wave number, defined through the Fourier transform of $\Psi$ as

$$
k_{\Psi}=\frac{\int k|\hat{\Psi}(k)| d k}{\int|\hat{\Psi}(k)| d k} .
$$

As discussed in [13], this global wavelet spectrum converges towards the Fourier energy spectrum provided $\Psi$ has enough vanishing moments. Its advantage is that it is less noisy than the Fourier energy spectrum, since it can be interpreted as a moving average of the energy spectrum over the wave number space [13]. The wavelet spectrum is provided in Fig. 18 following a "universal" representation $E^{W}(\ell) / \epsilon^{2 / 3} / \eta^{5 / 3}=F(\eta / \ell)$, with $\ell=k_{\Psi} / k$, for the cases we considered in the paper. One sees that the superposition of the 5 cases follows a $\ell^{-5 / 3}$ law over a large interval of wave number, that can be interpreted as an inertial range. Case A corresponds to injection scales, cases B, $\mathrm{C}$, and $\mathrm{D}$ correspond to inertial range, while case $\mathrm{E}$ is in the dissipative range.

\section{Diagnostics for energy transfer and dissipation}

Given any instantaneous velocity field, we can compute the 2D-3C velocity increments $\delta \vec{u}(\vec{r})=\vec{u}\left(\vec{x}^{2 D}+\vec{r}^{2 D}\right)-\vec{u}\left(\vec{x}^{2 D}\right)$, $\vec{x}^{2 D}$, and $\vec{r}^{2 D}$ being the coordinates onto the plane of measurements. In this paper, for simplicity, we omit the ${ }^{2 D}$ superscript.

Following Eq. (10), we may then use our measurements to compute the quantity $\mathscr{D}_{\ell}^{\mathrm{I}}$ and $\mathscr{D}_{\ell}^{\nu}$ :

$$
\begin{aligned}
& \mathscr{D}_{\ell}^{\mathrm{I}}=\frac{1}{4} \int \nabla \phi^{\ell}(\xi) \cdot \delta \boldsymbol{u}(\delta u)^{2}, \\
& \mathscr{D}_{\ell}^{v}=-v \int \nabla^{2} \phi^{\ell}(\xi) \boldsymbol{u}(x) \cdot \boldsymbol{u}(x+\xi) d \xi .
\end{aligned}
$$



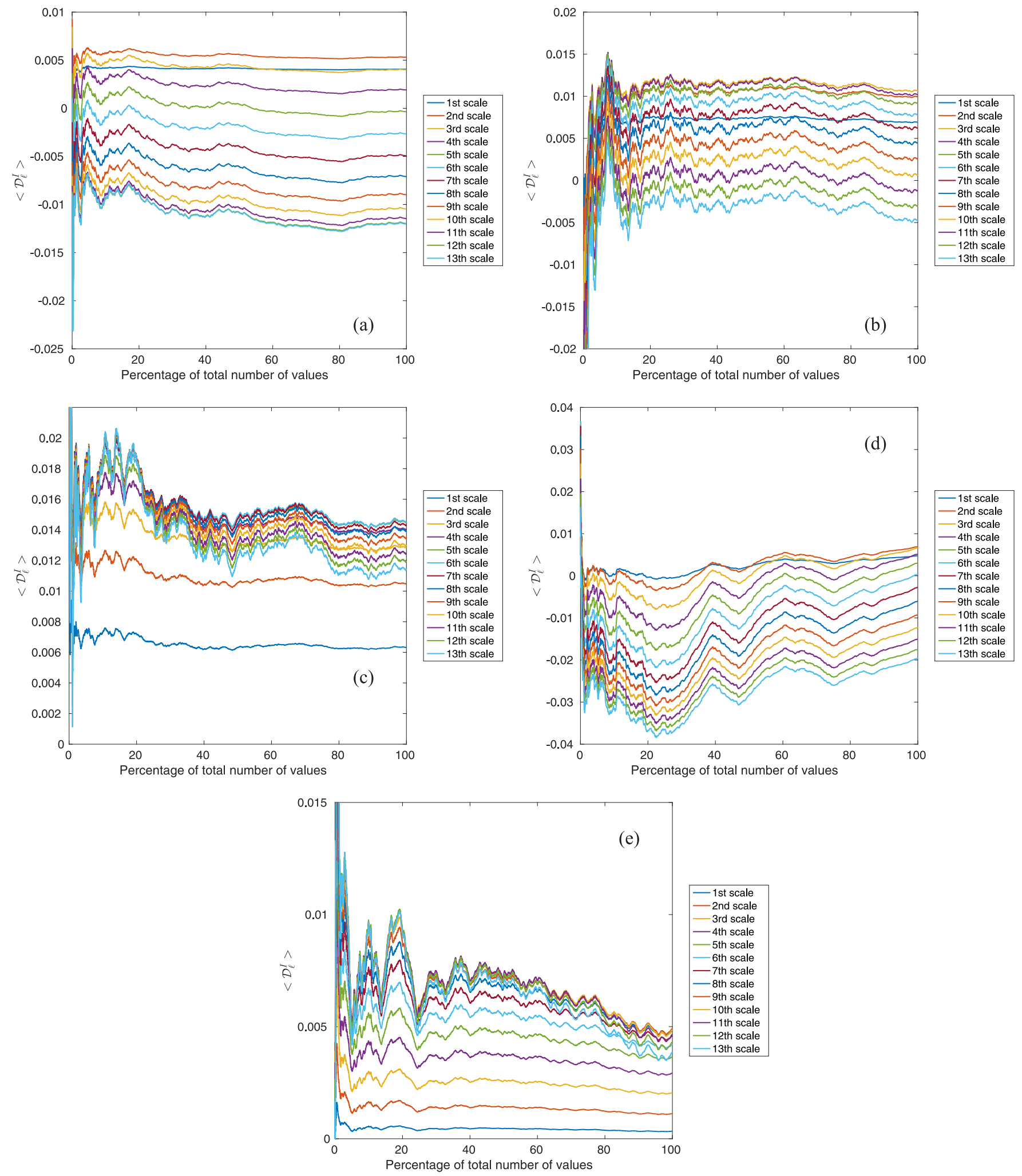

FIG. 19. Convergence of $\mathscr{D}_{\ell}^{\mathrm{I}}$ for cases A (a), B (b), C (c), D (d), and E (e).

For this, we use a simple shift in the definition of $\mathscr{D}_{\ell}^{v}$ to write it in term of a continuous wavelet transform as

$$
\mathscr{D}_{\ell}^{v}(\boldsymbol{x})=-v u_{i}(\boldsymbol{x}) W T_{i}(\Psi, \boldsymbol{u}, \ell, \boldsymbol{x}),
$$

where $W T_{i}(\Psi, \boldsymbol{u}, \ell, \boldsymbol{x})$ is the continuous wavelet transform of $u_{i}$ with respect to the wavelet $\Psi=\nabla^{2} \phi$. Taking $\phi$ as a Gaussian transforms $\Psi$ into the Mexican hat wavelet. The advantage of such a formulation is that it transforms the problem of computation of $\mathscr{D}_{\ell}^{v}(\boldsymbol{x})$ into the problem of computing 3 continuous wavelet transform, which is very fast using algorithms based on FFT. In the sequel, we use the 2D continuous wavelet MATLAB package provided by the toolbox YAWTB [35]. 

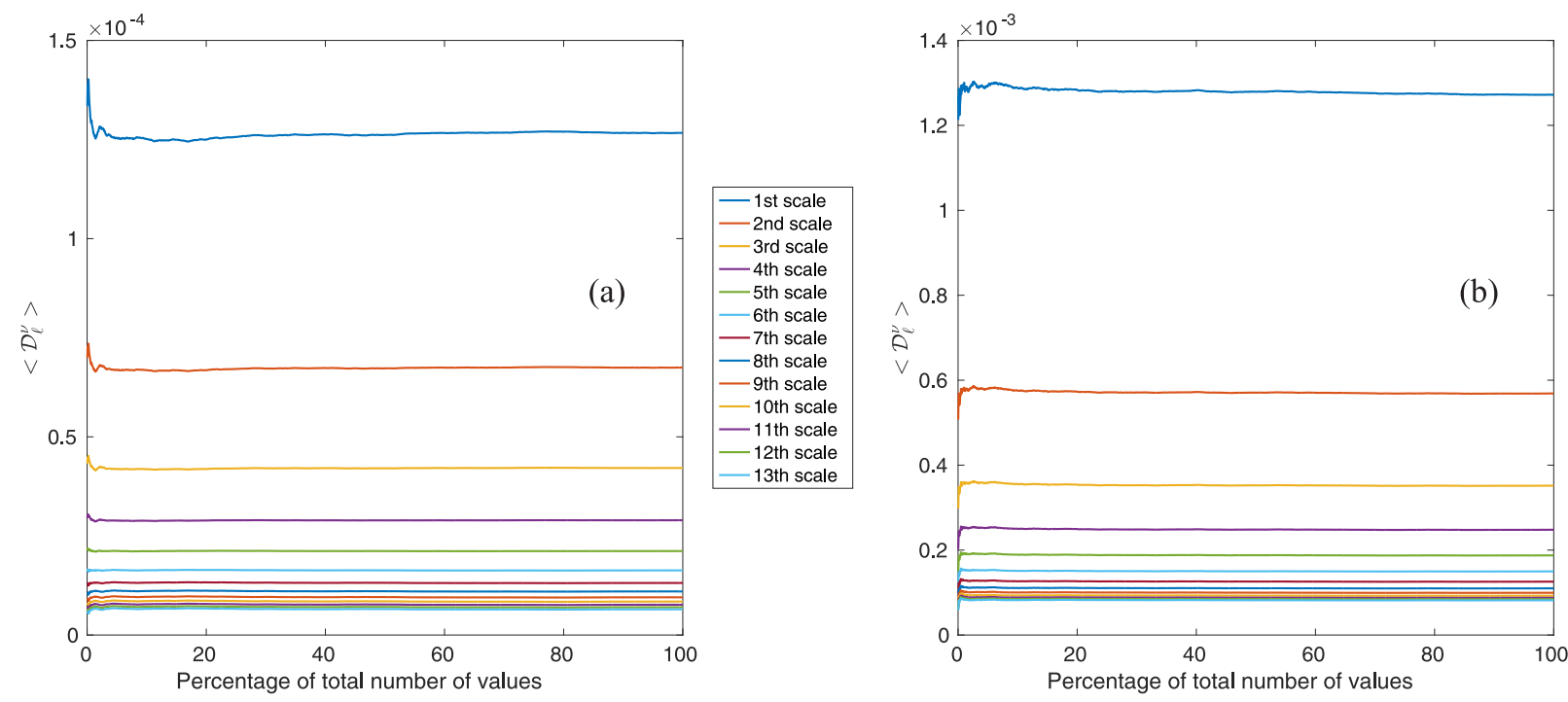

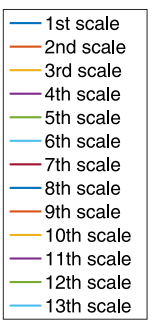
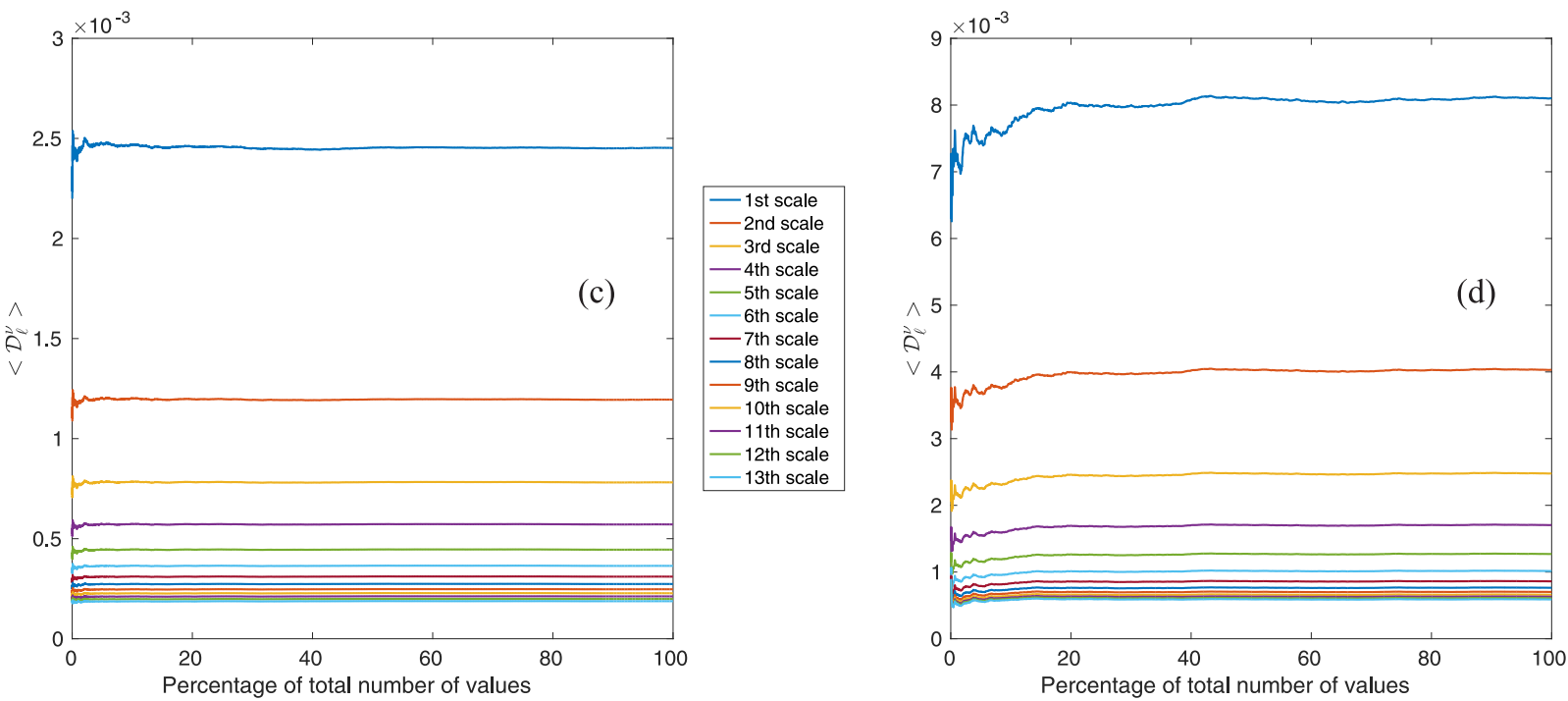

\begin{tabular}{|l|}
\hline -1st scale \\
-2nd scale \\
-3rd scale \\
-4th scale \\
- 5th scale \\
6th scale \\
- 7th scale \\
- 8th scale \\
- 9th scale \\
10th scale \\
- 11th scale \\
- 12th scale \\
13th scale \\
\hline
\end{tabular}

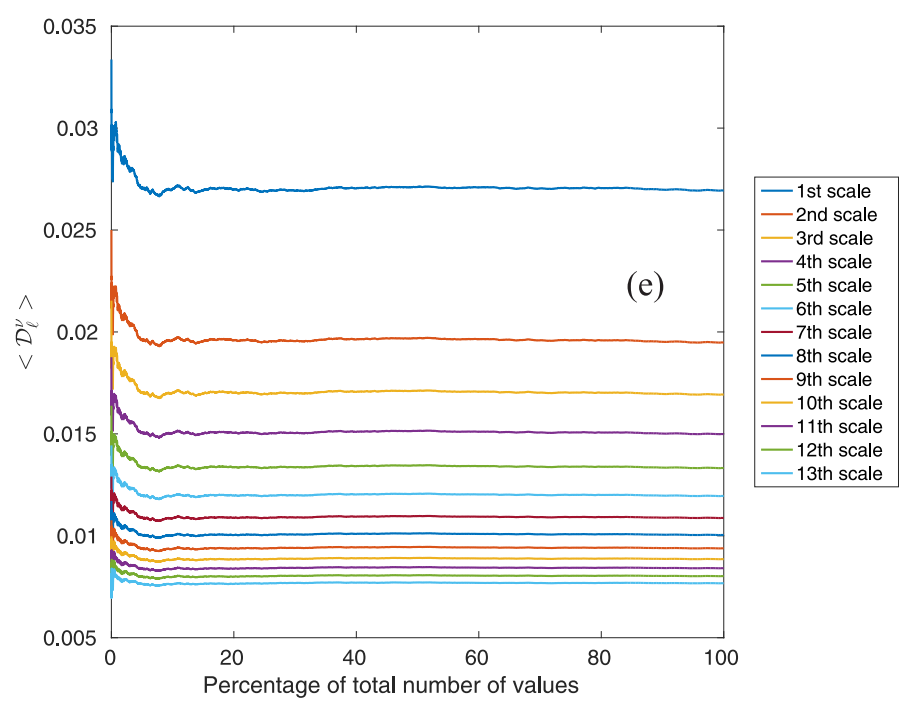

FIG. 20. Convergence of $\mathscr{D}_{\ell}^{v}$ for cases A (a), B (b), C (c), D (d), and E (e). 

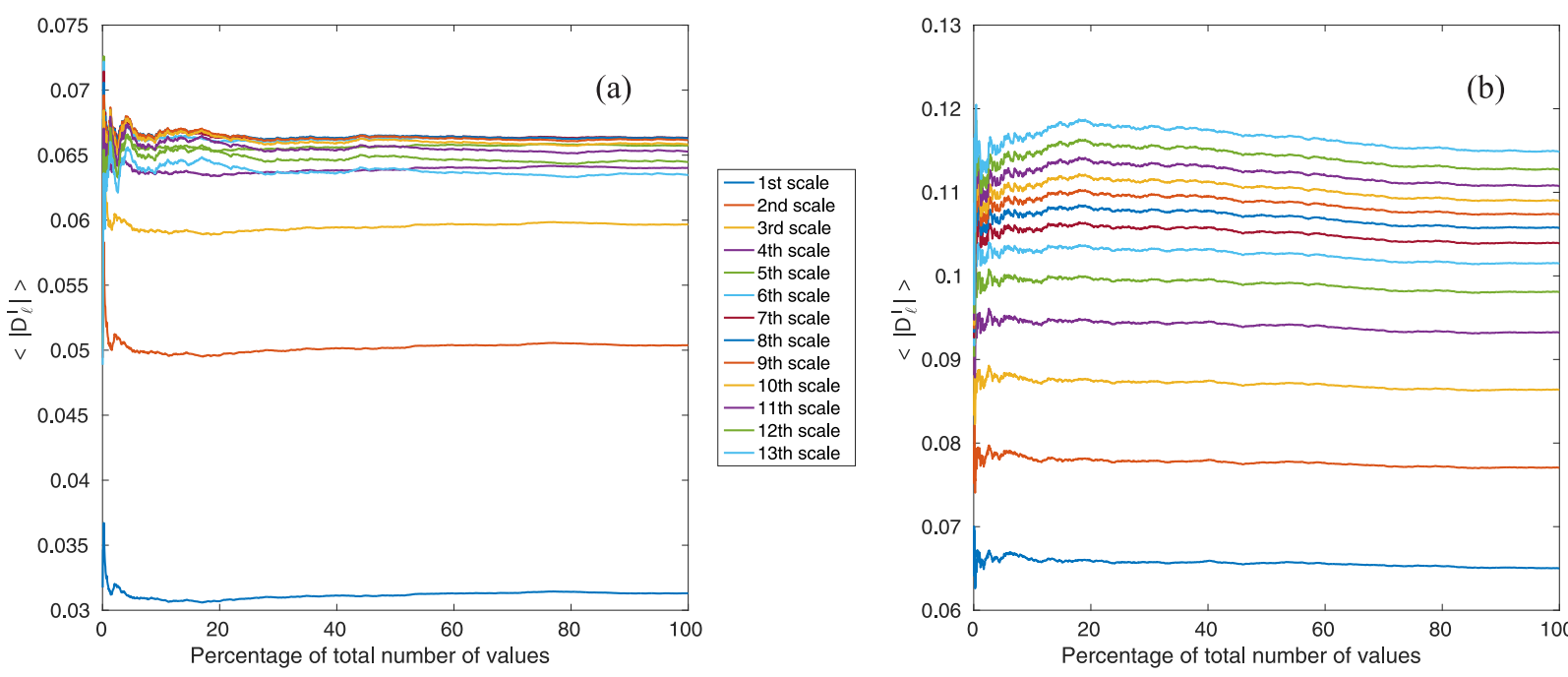

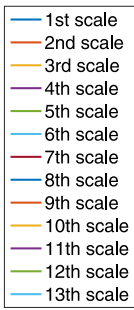
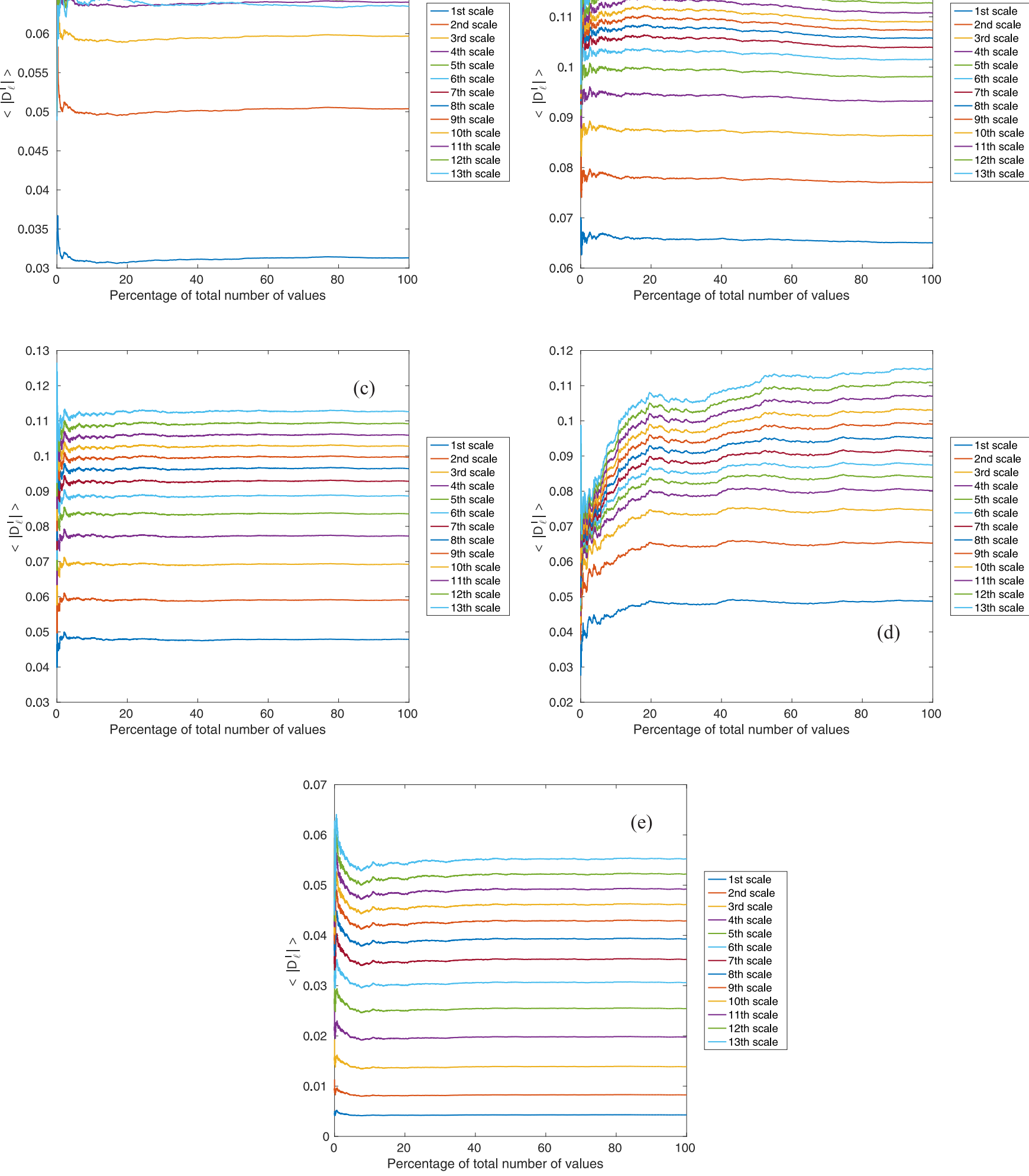

FIG. 21. Convergence of $\left|\mathscr{D}_{\ell}^{\mathrm{I}}\right|$ for cases A (a), B (b), C (c), D (d), and E (e). 
In the same way, one can use continuous wavelet transforms to compute efficiently $\mathscr{D}_{\ell}^{\mathrm{I}}$. Indeed, developing its expression, we get 18 terms looking typically like

$$
T_{11}=\frac{1}{4} \int \nabla_{1} \phi^{\ell}(\xi) u_{1}(x+\xi)|u(x+\xi)|^{2},
$$

which can be expressed as

$$
T_{11}=\frac{1}{4} W T_{1}\left(\nabla_{1} \phi, u|u|^{2}, \ell, \boldsymbol{x}\right) .
$$

Using complex wave numbers and complex wavelet transforms, we can then compute the whole term $\mathscr{D}_{\ell}^{\mathrm{I}}$ using only 9 complex continuous wavelet transforms.

For the DNS, we have used the same method, using the 3D continuous wavelet MATLAB package provided by the toolbox YAWTB.

\section{Convergency analysis}

To check the convergency of the quantity $\mathscr{D}_{\ell}^{\mathrm{I}}$ and $\mathscr{D}_{\ell}^{v}$, we have computed the running average of a variable ensemble of velocity fields, ranging from $0 \%$ of the total number of frames to $100 \%$ of the total number. The results are displayed in Figs. 19 and 20. One sees that the average is converged in all cases and for all scales for $\mathscr{D}_{\ell}^{v}$, and only for the first scales for the high-resolution data set $\mathrm{C}$ and $\mathrm{E}$ for $\mathscr{D}_{\ell}^{\mathrm{I}}$. For all other cases and scales, the average of $\mathscr{D}_{\ell}^{\mathrm{I}}$ is not converged within our statistics. If one looks for the average of the absolute value of $\mathscr{D}_{\ell}^{\mathrm{I}}$, the convergency is ensured for all cases and scales, as can be seen in Fig. 21.

\section{Generalized Pareto distribution analysis}

To perform the extreme value analysis we have chosen the so-called peak-over-threshold approach formalized by Pickands [34]. In this approach one considers a series of independent and identically distributed variables $X_{1}, X_{2}, \ldots, X_{n}$ and studies the probability $P(X>t)$ of exceeding a threshold $t$ corresponding to a large quantile. The Pickands theorem states that such exceedings asymptotically obey a generalized Pareto distribution (GPD) distribution with cumulative distribution function:

$$
F_{G}(x ; \mu, \sigma, \xi)=1-\left[1+\xi\left(\frac{x-\mu}{\sigma}\right)\right]^{-1 / \xi},
$$

where $\mu \in \mathbb{R}$ is the location parameter representing the chosen threshold and $\sigma>0$ is the scale parameter, representing the typical order of extreme fluctuations. The sign of $\xi$ discriminates the kind of tail decay of the parent distribution: When $\xi=0$, the distribution is of Gumbel type with exponentially decaying tail and Eq. (A9) reduces to

$$
F_{G}(x ; \mu, \sigma, \xi)=1-\exp \left(-\frac{x-\mu}{\sigma}\right) .
$$

The case $\xi>0$, corresponds to a Fréchet distribution with a fat tail decaying as a power law. Conversely, the case $\xi<0$ corresponds to the Weibull distribution with a bounded tail. In the Fréchet $\xi>0$ case, only the moments up to $1 / \xi$ exist, meaning that there is a nonzero probability of observing infinite values for the observable analyzed. The parameters are estimated using the MATLAB function gpfit that use a maximum likelihood estimator.

\section{Direct numerical simulations: Initial data and forcing}

To start the DNS run, we use the Taylor-Green initial velocity field given by

$$
\begin{aligned}
& u_{x}=\sin (x) \cos (y) \cos (z), \\
& u_{y}=-\cos (x) \sin (y) \cos (z), \\
& u_{z}=0 .
\end{aligned}
$$

We obtain turbulent steady states of the INSE by using the Taylor-Green forcing:

$$
\begin{aligned}
& f_{x}=f_{0} \sin \left(\tilde{k}_{\mathrm{f}} x\right) \cos \left(\tilde{k}_{\mathrm{f}} y\right) \cos \left(\tilde{k}_{\mathrm{f}} z\right), \\
& f_{y}=-f_{0} \cos \left(\tilde{k}_{\mathrm{f}} x\right) \sin \left(\tilde{k}_{\mathrm{f}} y\right) \cos \left(\tilde{k}_{\mathrm{f}} z\right), \\
& f_{z}=0,
\end{aligned}
$$

where $f_{0}$ and $\tilde{k}_{\mathrm{f}}$ are the forcing amplitude and wave number, respectively. We define $k_{\mathrm{f}}=\sqrt{3} \tilde{k}_{\mathrm{f}}$ as the amplitude of the forcing wave vector $\mathbf{k}_{\mathrm{f}}=\left(\tilde{k}_{\mathrm{f}}, \tilde{k}_{\mathrm{f}}, \tilde{k}_{\mathrm{f}}\right)$.
[1] A. N. Kolmogorov, Dokl. Akad. Nauk SSSR 30, 301 (1941).

[2] A. N. Kolmogorov, J. Fluid Mech. 13, 82 (1962).

[3] A. M. Oboukhov, J. Fluid Mech. 13, 77 (1962).

[4] R. H. Kraichnan, J. Fluid Mech. 62, 305 (1974).

[5] C. Meneveau, J. Fluid Mech. 232, 469 (1991).

[6] G. L. Eyink, J. Stat. Phys. 78, 335 (1995).

[7] L. Onsager, Il Nuovo Cimento 6, 279 (1949).

[8] U. Frisch, Turbulence: The Legacy of A. N. Kolmogorov (Cambridge University Press, Cambridge, 1995).

[9] G. L. Eyink, J. Stat. Phys. 78, 353 (1995).

[10] U. Frisch and G. Parisi, in Turbulence and Predictability in Geophysical Fluid Dynamics and Climate Dynamics, edited by M. Gil, R. Benzi, and G. Parisi (Elsevier, Amsterdam, 1985), pp. $84-88$.

[11] J. Duchon and R. Robert, Nonlinearity 13, 249 (2000).
[12] G. L. Eyink, Turbulence Theory, course notes, The Johns Hopkins University, 2007-2008, http://www.ams.jhu.edu/ eyink/ Turbulence/notes/ChapterIIIb.pdf.

[13] M. Farge and K. Schneider, https://www2.warwick.ac.uk/fac/ sci/physics/research/cfsa/people/sandrac/lectures/farge _wavelets_turb2002.pdf.

[14] T. D. Drivas and G. L. Eyink, Commun. Math. Phys. 359, 733 (2018).

[15] A. A. Mailybaev, Phys. Rev. E 87, 053011 (2013).

[16] E. W. Saw et al., Nat. Commun. 7, 12466 (2016).

[17] E. W. Saw et al., J. Fluid Mech. 837, 657 (2018).

[18] J. F. Pinton and R. Labbé, J. Phys. II 4, 1461 (1994).

[19] G. Zocchi, P. Tabeling, J. Maurer, and H. Willaime, Phys. Rev. E 50, 3693 (1994).

[20] P. Kestener and A. Arneodo, Phys. Rev. Lett. 93, 044501 (2004). 
[21] A. Arneodo et al., Europhys. Lett. 34, 411 (1996).

[22] D. Kuzzay et al., Nonlinearity 30, 2381 (2017).

[23] B. Dubrulle, Phys. Rev. Lett. 73, 959 (1994).

[24] J. F. Muzy, E. Bacry, and A. Kozhemyak, Phys. Rev. E 73, 066114 (2006).

[25] P. Abry, V. Pipiras, and H. Wendt, in Proc. GRETSI Symposium Signal and Image Processing, Troyes, France (GRETSI, 2007).

[26] B. Castaing, J. Phys. 50, 147 (1989).

[27] B. Castaing, Y. Gagne, and E. J. Hopfinger, Phys. D (Amsterdam, Neth.) 46, 177 (1990).

[28] B. Dubrulle and F. Graner, Phys. Rev. E 56, 6435 (1997).

[29] M. R. Leadbetter, G. Lindgren, and H. Rootzén, Extremes and Related Properties of Random Sequences and Processes
(Springer Science \& Business Media, New York, Berlin, Heidelberg, 2012).

[30] D. Faranda, V. Lembo, M. Iyer, D. Kuzzay, S. Chibbaro, F. Daviaud, and B. Dubrulle, J. Atmos. Sci. (2018), doi:10.1175/JASD-17-0114.1.

[31] R. H. Kraichnan, Adv. Math. 16, 305 (1975).

[32] B. Saint-Michel et al., Phys. Fluids 26, 125109 (2014).

[33] F. Ravelet, A. Chiffaudel, and F. Daviaud, J. Fluid Mech. 601, 339 (2008).

[34] J. Pickands, Ann. Stat. 3, 119 (1975).

[35] The toolbox YAWTB is available freely at http://sites.uclouvain.be/ispgroup/yawtb 